\title{
On the oscillatory hydrodynamic modes in liquid metal layers with an obstruction located on the bottom
}

\author{
Marcello Lappa \\ Department of Mechanical and Aerospace Engineering, University of Strathclyde \\ James Weir Building, 75 Montrose Street, Glasgow, G1 1XJ, UK \\ email: marcello.lappa@strath.ac.uk-marlappa@unina.it
}

\begin{abstract}
Hydrodynamic disturbances represent the preferred mode of instability of thermogravitational flow for a relatively wide range of substances and conditions (essentially pure or compound semiconductor and superconductor materials in liquid state). As nowadays almost all modern technologies rely greatly on such crystallized materials, targeting an improved understanding of the convective phenomena which occur in the melt has become a subject of great importance. Here an "ad hoc" model is developed to inquire specifically about the role played in such a context by geometrical "irregularities" affecting the melt container. More precisely, results are presented for the case of a fluid with $\mathrm{Pr}=0.01$ (silicon) filling an open cavity with a single backward-facing or forward-facing step on the bottom wall or with an obstruction located in the centre. It is shown that the presence of sudden changes in the considered geometry can lead to a variety of scenarios with a significant departure from classical situations examined in the past. These configurations have different spatial symmetries and show different dynamics, including rhythmic roll expansions and contractions along the vertical and horizontal directions at different locations, roll nucleation, deformation, transport and merging phenomena. In some circumstances a travelling wave with front perpendicular to the imposed temperature gradient emerges, which has never been reported in the literature. A frequency spectrum analysis is used to support the identification of the multiple convective phenomena enabled by the new geometric features.
\end{abstract}

Keywords: Liquid metal, Hadley flow, hydrodynamic disturbances, backward-facing or forwardfacing step.

\section{Introduction}

Over the past thirty or forty years, the industrial world has witnessed the rapid development of new technologies based on a variety of pure or compound semiconductor and superconductor crystals. Such crystals constitute the basis for microprocessors and computer memories and play an important role in several related advanced applications.

Most remarkably, although much effort has been directed to the technological development of such materials, current attempts towards further "improvements" (in terms of "product" quality and production costs) seem to show no obvious sign of reaching a stop. Indeed, with the growing deployment of such crystalline substances in new fields and for innovative industrial exploitations, researchers continue to keep concentrated on these subjects. In particular, as the manufacturing technology becomes increasingly more mature and standardized, and few uncertainties are left in the physical and chemical "overarching" factors which determine the performances of such materials in the solid state, "optimization" of related production techniques from a "fluid-dynamic" perspective is becoming a topic of considerable interest. 
Industrial methods for which such an optimization is being actively sought include the horizontal Bridgman (HB), the Floating zone (FZ) or the Czochralski (CZ) technique (see, e.g., [1,2]). A feature common to all such processes is the presence of thermal (or compositional) gradients in the melt, which, in general, provide driving forces for natural convection. The melt is, therefore, subject to non-uniform heat- and mass-transfer conditions, which often result in strongly undesired effects.

Of special interest in such a context is the so-called "Hadley flow", namely buoyancy convection driven by horizontal temperature gradients in elongated cavities or shallow geometries (Dupret and Van der Bogaert [3]; Monberg [4]). Since the pioneering work of Hadley [5] (who originally introduced this flow model as a simplified representation of the circulation developing in our atmosphere), such regimes of fluid motion have been largely studied due to their relevance to the industrial processes mentioned above. In particular, Hurle [6] was the first to show that oscillatory buoyancy convection of this type could be responsible for the emergence of undesired "imperfections" in crystallized materials.

The nature and spatio-temporal structure of the fluid-dynamic disturbances responsible for such oscillations were clarified in ensuing studies such as those by Hart [7,8] and Gill [9]. These authors identified two main categories of disturbances according to their spatial structure (transverse and longitudinal). They also disclosed that the so-called transversal instability is driven by the mean shear stress (this is the reason why it is often referred to as "shear instability" and the related disturbances as hydrodynamic ones), whereas the longitudinal instability arises as a consequence of a dynamical coupling between the mean shear stress and the thermal buoyancy force.

In the first case, the instability manifests itself in the form of two-dimensional (2D) vortices appearing on the frontier of the two opposing (primary and return) horizontal currents characterizing the basic flow. For open cavities (upper free surface exposed to an ambient gas) such disturbances are in general oscillatory (the first instability is a Hopf bifurcation, Laure and Roux [10]; Wang and Korpela [11]; Okada and Ozoe [12]; Gelfgat et al. [13]), whereas for closed cavities the Hopf bifurcation is generally preceded by a stationary instability. Regardless of their stationary or oscillatory nature, these vortices have axis perpendicular to the imposed temperature gradient (the general outcome of this instability is the replacement of the initial "unicellular" Hadley flow with a two-dimensional multicellular convective structure). The other mode of convection due to longitudinal disturbances consists essentially of a pair of gravitational or "helical" waves traveling in the spanwise direction, i.e. with front parallel to the temperature gradient.

Such instabilities can be controlled and/or related effects "mitigated" via the application of magnetic fields (Ben Hadid et al.[14]; Kaddeche et al. [15-17]) or imposed vibrations (Lappa [18]). By contrast, the flow becomes increasingly more complex if the configuration is "inclined" with respect to vertical gravity (Delgado-Buscalioni et al. [19]) or if solid/liquid phase change is taking place (El Ganaoui et al. [20]; Le Quéré and Gobin [21]). Such past findings have been obtained essentially for very simple geometrical models such as layers of infinite extent or bounded shallow rectangular cavities (these simplified and "easy to handle" configurations have been expressly used by researchers, on the one hand, to get important fundamental information on these phenomena and, 
on the other hand, to introduce well-defined benchmark cases for model validation and comparison).

While for the companion case of buoyancy convection in gases, some effort has been provided to expand such a framework by considering (both experimentally and numerically) flows over vertical or horizontal backward- and forward-facing steps (Hong et al. [22]; Abu-Mulaweh et al. [23]; AbuMulaweh [24-26]; Meskini et al. [27]; Dihmani et al. [28]; Mahrouche et al. [29]), there seems to be a disappointing lack of equivalent knowledge relating to liquid metals or semiconductor melts.

For these substances, experimental works are rather rare, this being due in large part to the wellknown difficulties of setting up in a laboratory suitable test cases that would be at the same time well-controllable and allow exploration of a wide variety of dynamical regimes (a key observation regarding such a lack of results is that, in general, semiconductor materials are opaque and very reactive in the liquid state). Past geometrical models used for flow stability analyses usually contained a number of symmetries. The present investigation proceeds one step further with the express aim to devise a more general mathematical and numerical framework in which such simplifications are somehow removed and the results carefully diagnosed (a kind of "modelling hierarchy"). In this context, CFD has the potential to offer valuable insights. As a valid alternative to past analysis strategies based on difficult expensive experiments, recent advances in numerical techniques as well as computing hardware make sophisticated computer-aided modelling and simulations possible.

The layout of this paper is as follows. In Sect 2, a brief summary of existing theory for the considered class of fluid-dynamic disturbances is given. In this section we also lay the general foundation of our theoretical and mathematical treatment. The related numerical method is presented together with a mesh sensitivity analysis and a validation study. Section 3 is entirely devoted to the discussion of the structure and spatio-temporal behaviour of the emerging disturbances in connection with the specific geometrical details considered (geometries with varying cross-section, i.e. a topography in the bottom wall). Section 4 is used for a critical discussion of the present results in the light of past work devoted to flow instabilities in constrained geometries. In Section 5, this framework is further extended by examining the response of the system to the presence of surface-tension-driven effects introduced as "a weak perturbation" of the buoyancy flow (this subject complements that of pure hydrodynamic gravitational disturbances by addressing the case of "hybrid" convection, which so much attention has also received in the literature devoted to crystal growth from the melt in configuration with a free liquid-gas interface).

\section{Mathematical Model and Numerical Method}

\subsection{The System}

Following earlier studies, we concentrate on a two-dimensional shallow cavity with free liquid-gas interface parallel to the $\mathrm{x}$ axis and characteristic depth $\mathrm{d}$, laterally delimited by solid walls at different temperatures (one cooled, the other heated). 
This classical geometry (the so-called R-F system, Laure and Roux [10], see Fig. 1) is varied by incorporating in it a backward-facing or a forward-facing step. In order to characterize the resulting geometrical model, we introduce relevant non-dimensional parameters, namely: the overall system aspect ratio (A), defined as its length-to-depth ratio $(\mathrm{L} / \mathrm{d}), \Gamma=\mathrm{L}_{\mathrm{s}} / \mathrm{L}$ to account for the horizontal extension of the region with reduced cross-sectional area and $\Lambda=\mathrm{d}_{\mathrm{s}} / \mathrm{d}$, i.e. the nondimensional height or depth of the step (hereafter, simply referred to as the "expansion ratio" or "compression ratio" depending on the specific case considered). Combination of these two geometrical models results in a third configuration with an "obstruction" located in the centre (Fig. 1c) for which we introduce the additional parameter $\Gamma_{1}=\mathrm{L}_{1} / \mathrm{L}$ to account for the distance of the obstruction from the (cold) wall.
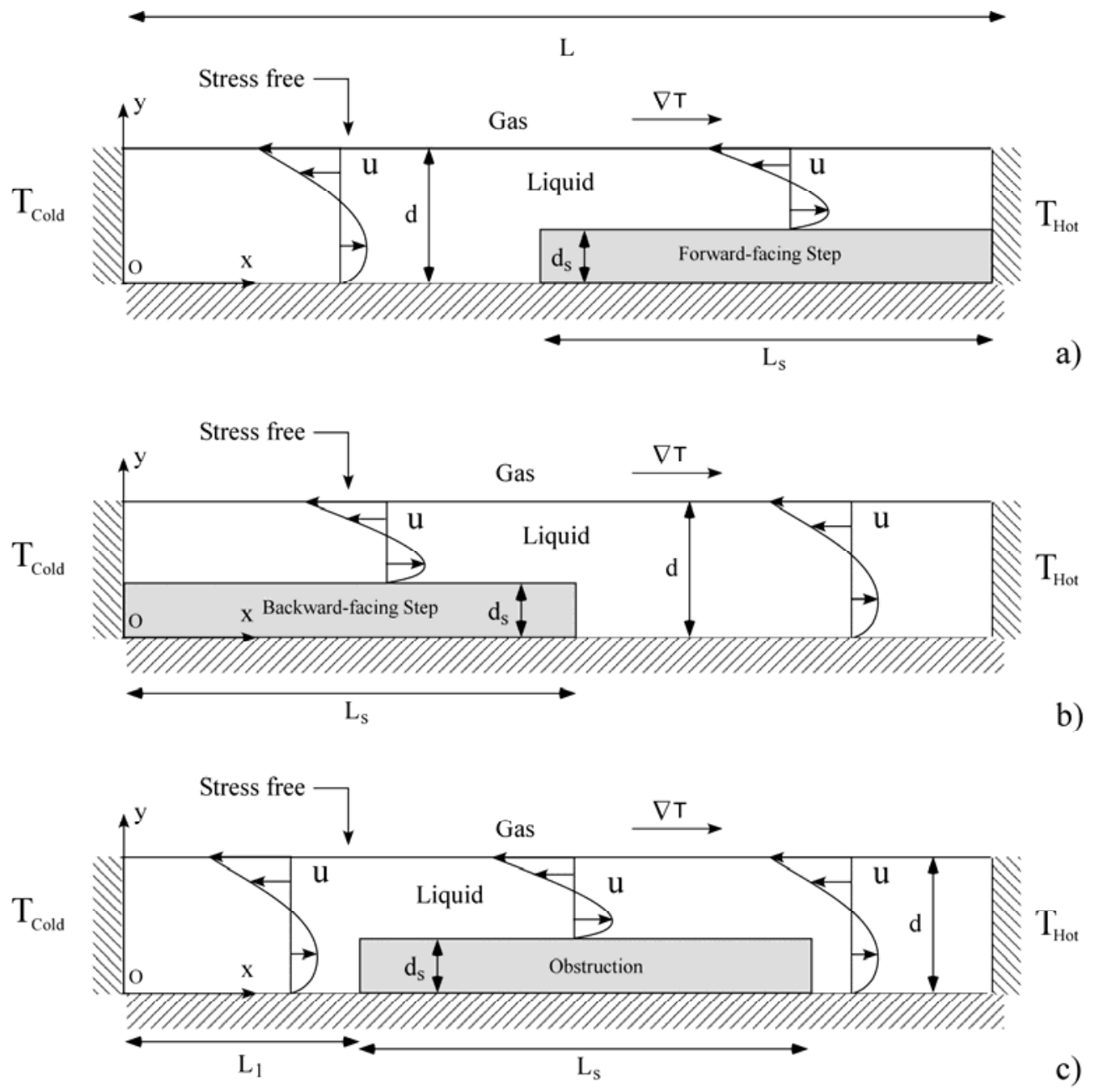

Figure 1: Sketch of the open cavity (so-called R-F configuration) supporting the emergence of hydrodynamic disturbances. The cavity can incorporate some geometrical variations.

\subsection{The considered fluid}

The aspect ratio of the cavity is fixed to $A=20$. The choice of a relevant work liquid to be considered accordingly is not as straightforward as one would expect. 
Indeed, both the nature of the fluid and the kinematic and thermal boundary conditions can play a significant role in determining the nature of the first instability (bifurcation) affecting the Hadley flow in such systems.

Some useful information along these lines (for the case of infinite or very elongated cavities such as that considered in the present work) can be extracted from the literature. As an example, in the case of top and bottom solid boundaries with adiabatic conditions, Hart [8] found transverse stationary hydrodynamic modes to be the most unstable if $\operatorname{Pr}<0.015$ (where $\operatorname{Pr}$ is the well-known Prandtl number: $v / \alpha, v$ being the fluid kinematic viscosity and $\alpha$ its thermal diffusivity), the longitudinal oscillating modes taking over in the range $0.015<\operatorname{Pr}<0.27$. These ranges were refined by Kuo and Korpela [30] and later by Laure and Roux [10], who extended these studies to rigid-free (R-F) configurations, confirming that hydrodynamic modes are the preferred mode of instability in the limit as $\operatorname{Pr} \rightarrow 0$ and that they become oscillatory when the upper wall is replaced by a free surface. Accordingly, we concentrate on silicon melt $(\mathrm{Pr}=0.01)$, this choice being motivated by the two-fold purpose of 1) considering a widely used semiconductor with diffused industrial relevance (most of microprocessors and computer memories are based on this substance) and 2) a melt expressly supporting the emergence of hydrodynamic disturbances of an oscillatory kind. Such disturbances are of a special interest to us because of their known ability to produce "variety" of dynamics even when the considered geometry is regular and symmetric (the reader being referred to the excellent numerical study by Gelfgat et al. [31] for additional details). Moreover, given the essentially twodimensional (2D) nature of these disturbances, one is allowed to perform the simulations in the framework of a 2D model, which makes this case an "ideal target" for a parametric investigation such as that performed here.

\subsection{The balance equations}

Using conventional reference quantities (see, e.g., [18,32], i.e. $d$ for the co-ordinates, $V_{\alpha}=\alpha / d, d^{2} / \alpha$ and $\rho \alpha^{2} / d^{2}$ (where $\rho$ is the fluid density), for velocity, time and pressure, respectively, and the classical Boussinesq approximation for liquids, the nondimensional momentum equation can be written as:

$$
\frac{\partial \underline{V}}{\partial}=-\underline{\nabla} p-\underline{\nabla} \cdot[\underline{V} \underline{V}]+\operatorname{Pr} \nabla^{2} \underline{V}+\operatorname{Pr} R a T \underline{i}_{g}
$$

where $\mathrm{Ra}$ is the well-known Rayleigh number defined as $\mathrm{Ra}=\mathrm{g} \beta_{\mathrm{T}} \Delta \mathrm{Td} / \nu \alpha\left(\beta_{\mathrm{T}}\right.$ being the thermal expansion coefficient and $\Delta \mathrm{T}$ the imposed temperature difference between the hot and cold sidewalls).

Accordingly, the continuity and energy equations can be cast in compact form as:

$\underline{\nabla} \cdot \underline{\mathrm{V}}=0$ 
and

$\frac{\partial T}{\partial t}=-\underline{\nabla} \cdot[\underline{V T}]+\nabla^{2} T$

where the nondimensional temperature has been defined as $T=\left(\bar{T}-\bar{T}_{\text {cold }}\right) / \Delta \mathrm{T}(\bar{T}$ is the dimensional temperature).

\subsection{The assumption of undeformable liquid-gas interface}

We assume the free liquid gas interface to be flat and undeformable. This assumption is not obvious and requires some additional physical reasoning and scaling analysis (based on the introduction of relevant non-dimensional parameters).

From a physical point of view there are different forces acting on such an interface. The first, of course, is gravity, which tends to render the free interface an equipotential surface, thereby making it perfectly horizontal (perpendicular to the gravity acceleration). The interface is also subjected to surface tension. This second force also tends to keep the interface flat and undeformable. Because the liquid in the cavity is not in quiescent conditions, a third category of forces exerting their influence on the interface is represented by the viscous forces produced by fluid motion (induced by buoyancy and/or Marangoni convection). The relative importance of all these effects can be measured by means of relevant non-dimensional (characteristic) numbers.

More specifically, the parameter accounting for the relative strength of viscous forces due to thermal convection and gravity is the so-called convective Galileo number:

$$
G a_{c}=\frac{\mu V_{r}}{\Delta \rho g d^{2}}
$$

where $\Delta \rho$ is the density difference between the considered liquid and air ( $\Delta \rho \cong \rho$ where $\rho$ is the melt density, see table I), $\mu$ is the melt dynamic viscosity and $V_{r}$ is a proper reference velocity and d has to be considered as the distance between the free surface and the bottom wall. Similarly, a parameter measuring the relative importance of viscous forces with respect to surface tension (the so-called Capillary number) can be introduced as:

$$
C a=\frac{\mu V_{r}}{\sigma}
$$

where $\sigma$ is the liquid surface tension. 


\begin{tabular}{||c|c||}
\hline Melting point temperature $\mathrm{T}_{\mathrm{m}}[\mathrm{K}]$ & 1685 \\
\hline Density $\rho\left[\mathrm{gr} / \mathrm{cm}^{3}\right]$ & 2.42 \\
\hline Thermal expansion coefficient $\beta_{\mathrm{T}}[1 / \mathrm{K}]$ & $1.4110^{-4}$ \\
\hline Surface tension $\sigma[\mathrm{dyne} / \mathrm{cm}]$ & $7.3310^{2}$ \\
\hline$\sigma_{\mathrm{T}}[$ dyne $/ \mathrm{cmK}]$ & $110^{-1}$ \\
\hline Thermal diffusivity $\alpha\left[\mathrm{cm}^{2} / \mathrm{s}\right]$ & $2.4410^{-1}$ \\
\hline Kinematic viscosity $v\left[\mathrm{~cm}^{2} / \mathrm{s}\right]$ & $2.4510^{-3}$ \\
\hline Prandtl number & 0.01 \\
\hline
\end{tabular}

Table I: Physical properties of Silicon melt

From the literature (see, e.g., [33-36]) it is known that when $G a_{c}<1$ or $C a<1$, the non-dimensional deformation $\delta$ experienced by a free equipotential surface under the action of viscous forces can be estimated in terms of order of magnitude as $\delta=\mathrm{O}\left(G a_{c}\right)$ or $\delta=\mathrm{O}(C a)$, respectively.

Because both gravity and surface tension play the role of "restoring" forces in the problem considered here (both tend to counteract the surface deformation dynamically induced by fluid convection), in the following we further split our analysis of the orders of magnitude into two parts, one being devoted to the case of gravity-dominated dynamics and another focusing on the condition in which surface tension becomes the prevailing restoring force. The relative importance of these two effects, in turn, can be estimated resorting to the so-called Bond number, generally defined in the literature as:

$$
B o=\frac{\Delta \rho g d^{2}}{\sigma} \cong \frac{\rho g d^{2}}{\sigma}
$$

Gravity and surface tension are equivalent when $B o=1$, which for the present conditions (see Table I) would be corresponding to a layer having a depth of $\cong 0.55 \mathrm{~cm}$.

First, we address the case $B o<1$ (dominant surface tension), i.e. layers with a relatively small depth $(<0.55 \mathrm{~cm})$.

In the case of buoyancy flow, the reference velocity $V_{r}$ must be obviously assumed to be the socalled buoyancy velocity

$$
V_{g}=\frac{g \beta_{T} \Delta T d^{2}}{v}
$$

The relevant parameter to be considered in this regime, namely the Capillary number, therefore reads

$$
C a=\frac{\rho g \beta_{T} \Delta T d^{2}}{\sigma}=\frac{B o R a}{\operatorname{Pr} G a}
$$

where $G a$ is the classical Galileo number $\left(\mathrm{gd}^{3} / \mathrm{v}^{2}\right)$. 
In the other limiting condition, in which gravity becomes the most significant restoring force $(d>0.55 \mathrm{~cm})$, the convective Galileo number, similarly, will read:

$$
G a_{c}=\frac{R a}{\operatorname{Pr} G a}
$$

The values taken by both the Capillary number and the convective Galileo number for the "worst" conditions considered in the present work in terms of viscous forces (namely $\mathrm{Ra}=15000$, see Sect. 3) can be seen in Table II:

\begin{tabular}{ccc} 
depth $[\mathrm{cm}]$ & $B o<1$ & $C a$ \\
\hline \hline $1 \times 10^{-2}$ & $3.24 \times 10^{-4}$ & 2.96 \\
$5 \times 10^{-2}$ & $8.10 \times 10^{-3}$ & $5.92 \times 10^{-1}$ \\
$1 \times 10^{-1}$ & $3.24 \times 10^{-2}$ & $2.96 \times 10^{-1}$ \\
$5 \times 10^{-1}$ & $8.10 \times 10^{-1}$ & $5.92 \times 10^{-2}$ \\
& & \\
depth $[\mathrm{cm}]$ & $B o>1$ & $G a_{c}$ \\
\hline \hline 1 & 3.24 & $9.14 \times 10^{-3}$ \\
5 & $8.10 \times 10$ & $7.31 \times 10^{-5}$ \\
10 & $3.24 \times 10^{2}$ & $9.14 \times 10^{-6}$ \\
50 & $8.10 \times 10^{3}$ & $7.31 \times 10^{-8}$ \\
100 & $3.24 \times 10^{4}$ & $9.14 \times 10^{-9}$ \\
500 & $8.10 \times 10^{5}$ & $7.31 \times 10^{-11}$
\end{tabular}

Table II: Bond number, Capillary number (Part a) and convective Galileo number (Part b) as a function of the layer depth for $\mathrm{Ra}=15000$.

Given the very small orders of magnitude of the Capillary and convective Galileo number evident in this table (because of the relatively small viscosity of liquid metals and their relatively high density and surface tension with respect to other (e.g., organic) liquids), it can therefore be concluded that for the considered fluid (silicon melt) the interface can be assumed flat and horizontal over a wide range of conditions (in practice, as shown in Table II, a lower boundary to the applicability of the present results is represented by the requirement that, in order to prevent the Capillary number from taking values $>\mathrm{O}\left(10^{-2}\right)$, the minimum distance between the free surface and the bottom wall should not be smaller than $0.5 \mathrm{~cm}$ ).

An additional check, however, is required for the case in which Marangoni convection is also present. The "worst conditions" in our case (see Sect. 5) correspond to $\mathrm{Ra}=15000$ and $\mathrm{Ma}=1000$, where Ma $=\sigma_{\mathrm{T}} \Delta \mathrm{Td} / \mu \alpha$ is the so-called Marangoni number $\left(\sigma_{\mathrm{T}}\right.$ being the surface tension derivative with respect to the temperature). Assuming the properties in Table I, this would be equivalent to considering a layer with depth $\cong 5 \mathrm{~cm}$ (for which $\mathrm{Ra}=15 \mathrm{Ma}$ ).

Replacing the buoyancy velocity $V_{g}$ appearing in the definition of the convective Galileo number with the corresponding reference velocity for Marangoni flow: 
$V_{M a}=\frac{\sigma_{T} \Delta T}{\mu}$

accordingly, the convective Galileo number can be re-cast in compact form as:

$$
G a_{c}=\frac{M a}{\operatorname{Pr} G a}
$$

The value taken by this parameter based on $V_{M a}$ for $\mathrm{Ma}=1000\left(\mathrm{Ga}_{\mathrm{c}}=4.5 \times 10^{-6}\right)$ and based on $V_{g}$ for $\mathrm{Ra}=15000\left(\mathrm{Ga}_{\mathrm{c}}=7.31 \times 10^{-5}\right)$ clearly indicates that for such conditions neither buoyancy convection, nor Marangoni flow are able to produc significant deformation of the free surface.

\subsection{The Initial and Boundary conditions}

As initial conditions we consider:

$\mathrm{t}=0: \underline{\mathrm{V}}(\mathrm{x}, \mathrm{y})=0, \mathrm{~T}(\mathrm{x}, \mathrm{y})=\mathrm{x} / \mathrm{A}$

i.e. the liquid is motionless with a linear temperature profile along the $\mathrm{x}$ coordinate (the temperature is $\mathrm{T}_{\text {Cold }}=0$ on the cold sidewall and $\mathrm{T}_{\mathrm{Hot}}=1$ on the other side). Adiabatic conditions are assumed on both horizontal boundaries. Accordingly, the boundary conditions read:

$\mathrm{u}=0, \mathrm{v}=0$ and $\mathrm{T}=0$ at $\mathrm{x}=0$ (left wall)

$\mathrm{u}=0, \mathrm{v}=0$ and $\mathrm{T}=1$ at $\mathrm{x}=\mathrm{A}$ (right wall)

$\mathrm{v}=0, \partial \mathrm{u} / \partial \mathrm{y}=-\mathrm{Ma} \partial \mathrm{T} / \partial \mathrm{x}$ and $\partial \mathrm{T} / \partial \mathrm{y}=0$ at $\mathrm{y}=1$ (free surface)

Equation (15) enforces a flow by tangential variation of the surface tension. Obviously, purely stress-free conditions, that is pure buoyancy flow, can be recovered by simply setting $\mathrm{Ma}=0$.

An additional useful non-dimensional parameter measuring the relative importance of surfacetension driven and buoyancy effects can be introduced as:

$\frac{M a}{R a}=W=\frac{\sigma_{T}}{g \rho \beta_{T}} L^{-2}$
$W=B_{d}^{-1}$

where $B_{d}$ is the well-known dynamic Bond number.

For the configuration with the backward-facing step, the following additional boundary conditions must be taken into account: 
$\mathrm{u}=0, \mathrm{v}=0$ at $\mathrm{y}=\Lambda$ for $0 \leq \mathrm{x} \leq \mathrm{A} \Gamma$

$\mathrm{u}=0, \mathrm{v}=0$ at $\mathrm{x}=\mathrm{A} \Gamma$ for $0 \leq \mathrm{y} \leq \Lambda$

$\mathrm{u}=0, \mathrm{v}=0$ at $\mathrm{y}=0$ for $\mathrm{A}(1-\Gamma) \leq \mathrm{x} \leq \mathrm{A}$

and (adiabatic bottom wall)

$\partial \mathrm{T} / \partial \mathrm{y}=0$ at $\mathrm{y}=\Lambda$ for $0 \leq \mathrm{x} \leq \mathrm{A} \Gamma$

$\partial \mathrm{T} / \partial \mathrm{x}=0$ at $\mathrm{x}=\mathrm{A} \Gamma$ for $0 \leq \mathrm{y} \leq \Lambda$

$\partial \mathrm{T} / \partial \mathrm{y}=0$ at $\mathrm{y}=0$ for $\mathrm{A}(1-\Gamma) \leq \mathrm{x} \leq \mathrm{A}$

For the configuration with the forward-facing step:

$\mathrm{u}=0, \mathrm{v}=0$ at $\mathrm{y}=0$ for $0 \leq \mathrm{x} \leq \mathrm{A}(1-\Gamma)$

$\mathrm{u}=0, \mathrm{v}=0$ at $\mathrm{x}=\mathrm{A}(1-\Gamma)$ for $0 \leq \mathrm{y} \leq \Lambda$

$\mathrm{u}=0, \mathrm{v}=0$ at $\mathrm{y}=\Lambda$ for $\mathrm{A}(1-\Gamma) \leq \mathrm{x} \leq \mathrm{A}$

and (adiabatic bottom wall)

$\partial \mathrm{T} / \partial \mathrm{y}=0$ at $\mathrm{y}=0$ for $0 \leq \mathrm{x} \leq \mathrm{A}(1-\Gamma)$

$\partial \mathrm{T} / \partial \mathrm{x}=0$ at $\mathrm{x}=\mathrm{A}(1-\Gamma)$ for $0 \leq \mathrm{y} \leq \Lambda$

$\partial \mathrm{T} / \partial \mathrm{y}=0$ at $\mathrm{y}=\Lambda$ for $\mathrm{A}(1-\Gamma) \leq \mathrm{x} \leq \mathrm{A}$

For the configuration with the obstruction in the centre:

$\mathrm{u}=0, \mathrm{v}=0$ at $\mathrm{y}=0$ for $0 \leq \mathrm{x} \leq \mathrm{A} \Gamma_{1}$

$\mathrm{u}=0, \mathrm{v}=0$ at $\mathrm{x}=\mathrm{A} \Gamma_{1}$ for $0 \leq \mathrm{y} \leq \Lambda$

$\mathrm{u}=0, \mathrm{v}=0$ at $\mathrm{y}=\Lambda$ for $\mathrm{A} \Gamma_{1} \leq \mathrm{x} \leq \mathrm{A}\left(\Gamma+\Gamma_{1}\right)$

$\mathrm{u}=0, \mathrm{v}=0$ at $\mathrm{x}=\mathrm{A}\left(\Gamma+\Gamma_{1}\right)$ for $0 \leq \mathrm{y} \leq \Lambda$

$\mathrm{u}=0, \mathrm{v}=0$ at $\mathrm{y}=0$ for $\mathrm{A}\left(\Gamma+\Gamma_{1}\right) \leq \mathrm{x} \leq \mathrm{A}$

and (adiabatic bottom wall)

$\partial \mathrm{T} / \partial \mathrm{y}=0$ at $\mathrm{y}=0$ for $0 \leq \mathrm{x} \leq \mathrm{A} \Gamma_{1}$

$\partial \mathrm{T} / \partial \mathrm{x}=0$ at $\mathrm{x}=\mathrm{A} \Gamma_{1}$ for $0 \leq \mathrm{y} \leq \Lambda$

$\partial \mathrm{T} / \partial \mathrm{y}=0$ at $\mathrm{y}=\Lambda$ for $\mathrm{A} \Gamma_{1} \leq \mathrm{x} \leq \mathrm{A}\left(\Gamma+\Gamma_{1}\right)$

$\partial \mathrm{T} / \partial \mathrm{x}=0$ at $\mathrm{x}=\mathrm{A}\left(\Gamma+\Gamma_{1}\right)$ for $0 \leq \mathrm{y} \leq \Lambda$

$\partial \mathrm{T} / \partial \mathrm{y}=0$ at $\mathrm{y}=0$ for $\mathrm{A}\left(\Gamma+\Gamma_{1}\right) \leq \mathrm{x} \leq \mathrm{A}$

\subsection{The Numerical method}

Balance equations and related boundary conditions (1-3, 12-22) have been solved numerically by a time-explicit method (primitive-variable approach) relying on a structured mesh and fluid-dynamic variables collocated on the grid in a staggered arrangement. In particular, the resulting finite- 
difference method has been implemented on the basis of forward differences in time and centraldifferencing schemes in space (second order accurate). The related solution strategy is not illustrated in detail here, the interested reader being referred to various books in the literature for an exhaustive treatment (e.g., Fletcher [37]). Here we limit ourselves just to illustrating the main outcomes of the grid refinement study and the strategy that we had to implement to guarantee a proper resolution of all spatial and temporal scales involved in the considered phenomena.

In particular, the "a priori" grid tailoring study (carried out to quantify the improved resolution achievable by using more dens grids), has been conducted for $\operatorname{Pr}=0.01$ and $\mathrm{Ra}=10^{4}$, using the following sensitive parameters to check grid independency:

- $\mathrm{U}_{\max }$ : maximum velocity along $\mathrm{x}$ axis;

- $\mathrm{V}_{\max }$ : maximum velocity along y axis;

- $f$ : frequency value of measured temperature oscillation.

\begin{tabular}{ccccccc}
\hline \hline Grid $\mathrm{N}_{\mathrm{x}} \times \mathrm{N}_{\mathrm{y}}$ & $\mathrm{U}_{\max }$ & $\Delta \%$ & $\mathrm{~V}_{\max }$ & $\Delta \%$ & $\mathrm{f}$ & $\Delta \%$ \\
\hline \hline $150 \times 30$ & 10.8697 & - & 7.1617 & - & 0.155 & - \\
$200 \times 30$ & 10.3195 & 4.9 & 5.2881 & 0.559 & 260 \\
$300 \times 30$ & 10.3265 & $<<1$ & 5.2817 & 0.554 & $<<1$ \\
$400 \times 30$ & 10.3338 & $<<1$ & 5.1698 & 0.5405 & $\cong 1$ \\
$600 \times 30$ & 10.3446 & $<<1$ & 5.2512 & 0.5434 & $<<1$ \\
\hline \hline
\end{tabular}

Table III: Grid Refinement Study: maximum velocity along the $\mathrm{x}$ axis; maximum velocity along the $\mathrm{y}$ axis and frequency of measured temperature oscillations as a function of mesh resolution $\left(\operatorname{Pr}=0.01, \mathrm{~A}=20\right.$, no steps or obstacles, $\left.\mathrm{Ra}=1 \times 10^{4}\right)$.

As evident in Table III, assuming 30 points along the vertical direction, in the absence of steps or sudden variations of the cross-sectional area, the minimum mesh required to guarantee independence of the velocity field (and its oscillation frequency) from the used spatial resolution is 200 points along the horizontal direction. However, we conducted most of simulations with 600 points in the $\mathrm{x}$ direction in order to keep the ratio between the grid steps along $\mathrm{x}$ and $\mathrm{y}$ of unit order (to ensure proper resolution along the horizontal direction when obstacles of different kinds are placed inside the cavity).

\section{$\underline{2.7 \text { Code validation }}$}

The code has been validated through comparison with the landmark numerical results of Gelfgat et al. [13] for pure buoyancy flow in an open rectangular cavity with $A=10, \operatorname{Pr}=0.015$ and 
$\mathrm{Ra}=9.32 \times 10^{3}$. Using the same grid density that, according to the refinement study carried out in Sect. 2.5, guarantees a mesh-independent solution (i.e. at least 30 grid points per unit nondimensional length), we have obtained an oscillatory solution with angular frequency $\omega \cong 4.92$ matching with a reasonable approximation $(\cong 2 \%)$ the value $(4.83)$ reported by these authors (see Figure 2).

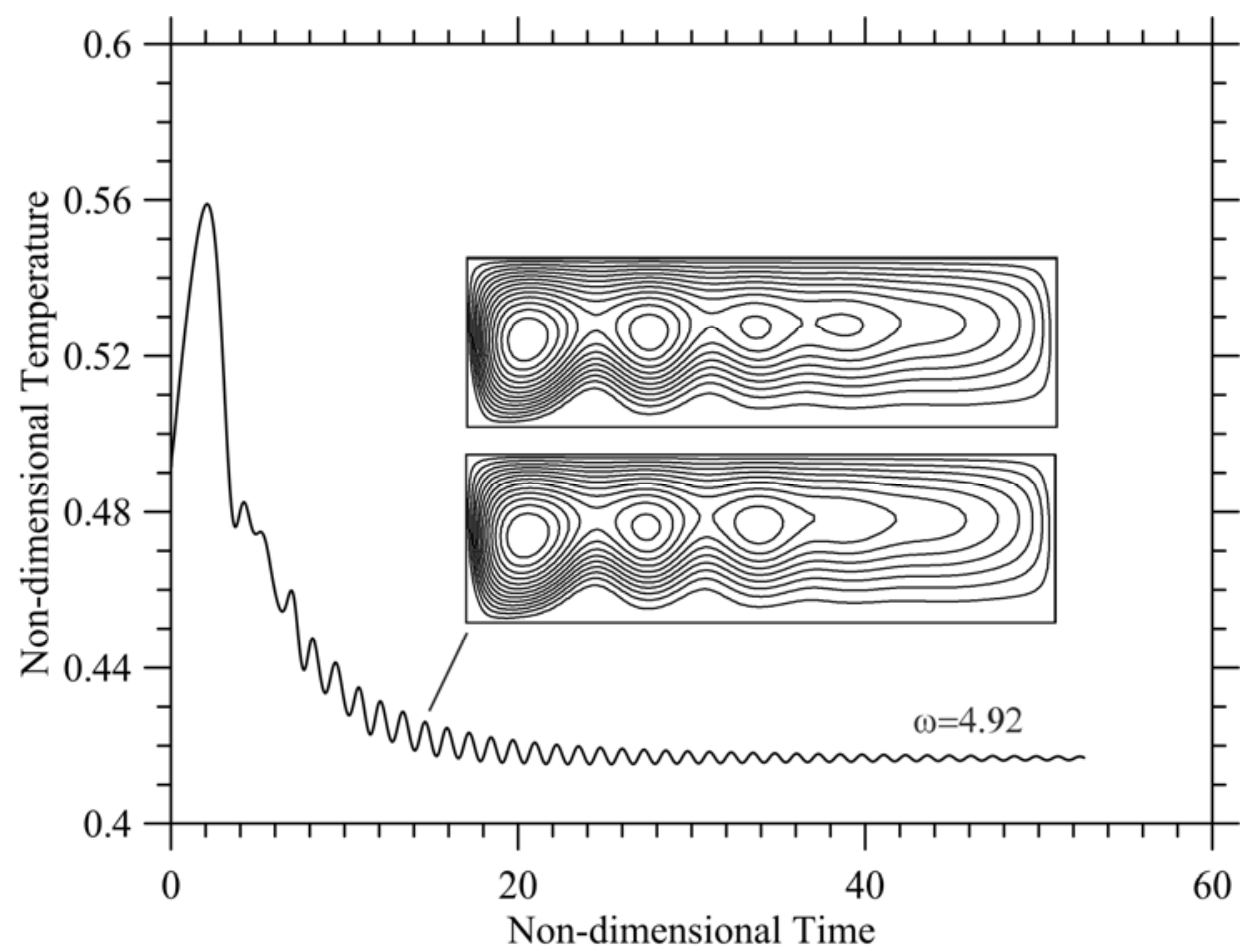

Figure 2: Comparison with the numerical results by Gelfgat et al. (1997): $\operatorname{Pr}=0.015, A=10$, $\mathrm{Ra}=9.3 \times 10^{3}$ (temperature signal measured at $\mathrm{x}=\mathrm{A} / 2$ in proximity to the free surface with two related snapshots of streamlines distribution) [for illustration purposes, in the figure above and in all the other figures reported in this work, the depth of the fluid layer has been increased by approximately two times with respect to its real dimension].

\section{Results}

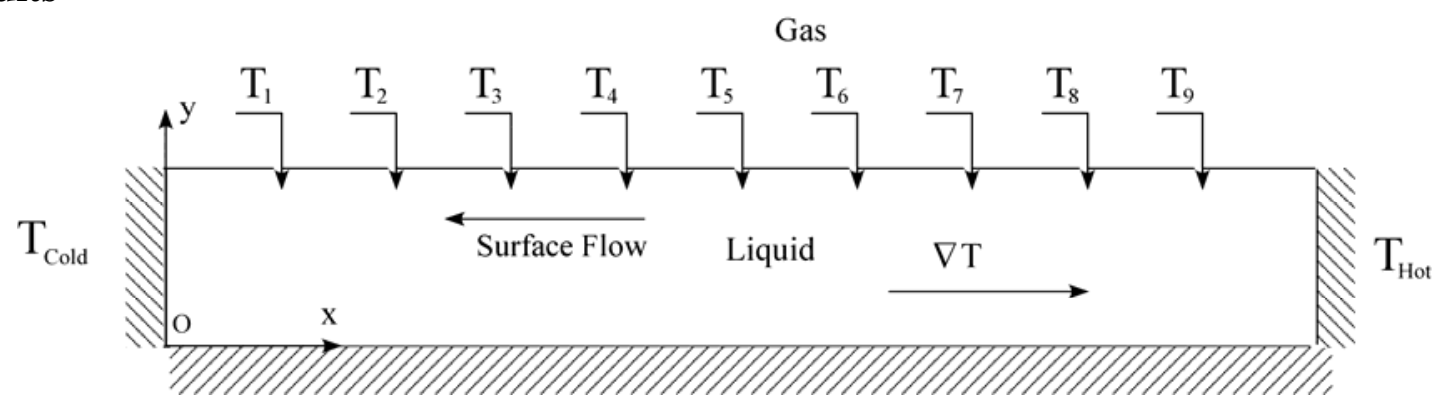

Figure 3: Distribution of numerical probes along the cavity.

For most of cases considered in the following, for illustration purposes, the behaviour of the temperature field as a function of time is expressly reported at different positions along the free liquid/gas interface. More specifically, we use nine "numerical probes", evenly spaced along the 
horizontal extension of the cavity as shown in Fig. 3. Obviously, relevant information is also provided in terms of streamlines distribution and snapshots of the velocity field at different times when the considered conditions support the development of oscillatory solutions. As anticipated in the introduction, we draw attention to a few features that do not seem to have received much attention in past studies.

\subsection{The classical rectangular open cavity}

Most conveniently, we start from the classical case with no steps or obstructions (canonical rectangular open cavity) to illustrate shortly the general property of the hydrodynamic mode, then the discussion progresses via comparison with the cases with a topography on the bottom wall (for illustration purposes, in all such figures the depth of the fluid layer is approximately two times its real dimension).

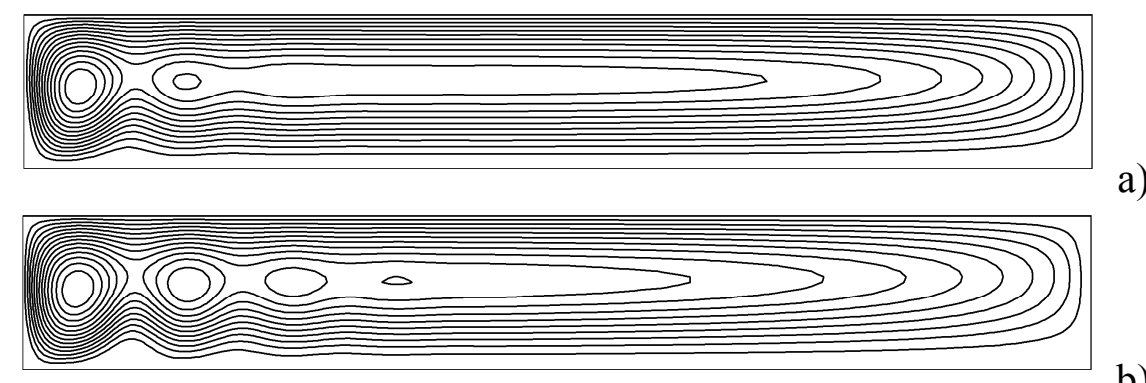

a)

\section{b)}

Figure 4: Streamlines $(\mathrm{Pr}=0.01, \mathrm{~A}=20)$ : a) $\mathrm{Ra}=2 \times 10^{3}$ (steady state, $\psi_{\max }=0.7$ ), b) $\mathrm{Ra}=5 \times 10^{3}$ (steady state, $\psi_{\max }=1.68$ ),

Figure 4 shows the emerging pattern for two different (subcritical) values of the Rayleigh number. The different rolls visible in the cavity are all part of a larger circulation system with fluid moving continuously from the hot side to the cold side along the free interface and then flowing back along the bottom wall. As the Rayleigh number increases, the number of distinguishable rolls embedded in the main circulation system also increases.

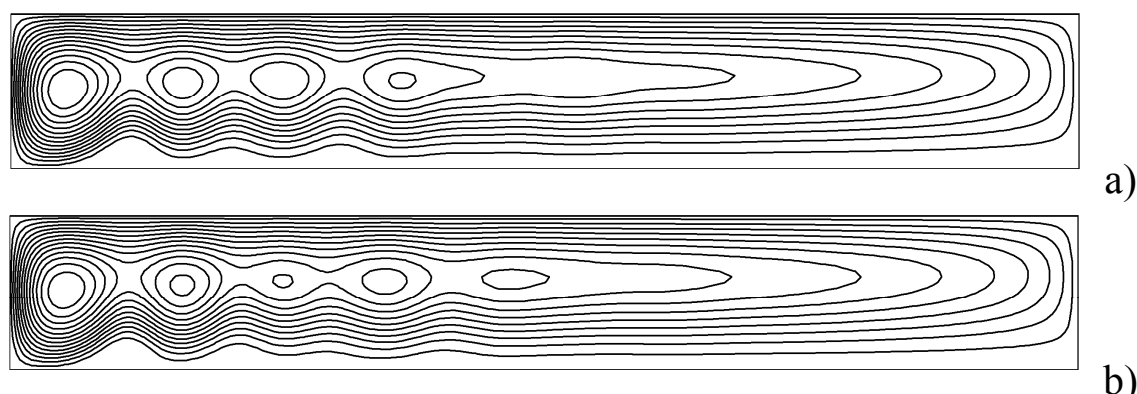

Figure 5: Streamlines $(\operatorname{Pr}=0.01, \mathrm{~A}=20): \mathrm{Ra}=1.5 \times 10^{4}$ (snapshots of oscillatory flow at two different times, $\psi_{\max }=3.9$ ). 
The behaviour completely changes, however, when the Rayleigh number is increased to $\mathrm{Ra}=$ $1.5 \times 10^{4}$. For this value of the Rayleigh number, the steady flow seen in Fig. 4 is completely replaced by a new mechanism with rolls at fixed locations which "pulsate" in time. (i.e. disturbance nodes growing and shrinking alternately in time undergoing a limited back and forth displacement in the streamwise direction, see Fig. 5). This is the typical feature of the oscillatory hydrodynamic mode, as also reported by other authors (e.g., [13,31]).

\subsection{Steady states: the effect of sudden changes in the geometry}

Sudden variations in the cross-sectional area of the considered geometry can lead to appreciable modifications of the flow even if it is still in subcritical conditions like those related to Fig. 4. This can be clearly seen in Figs. 6 and 7. Among other things, such figures lead to the almost immediate (and obvious) conclusion that the steady patterns emerging for the case of forward-facing or backward-facing steps are not the mirror image of one another.

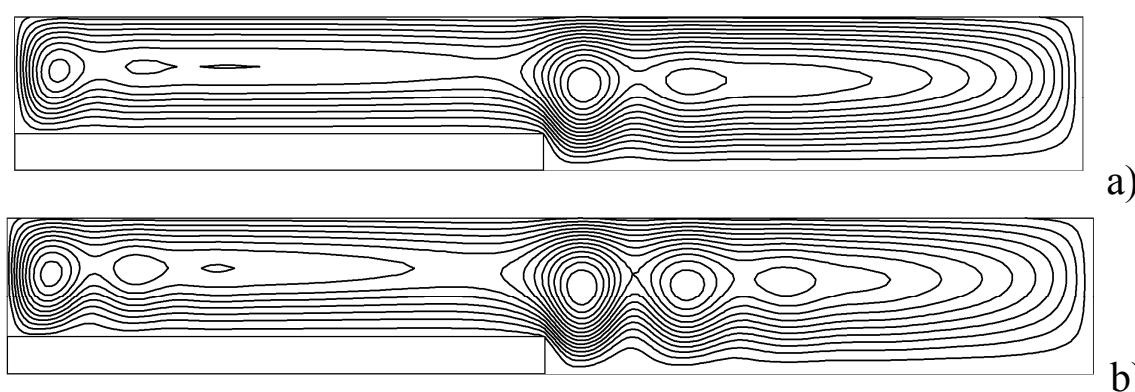

a)

b)

Figure 6: Streamlines ( $\operatorname{Pr}=0.01, A=20$, Backward-facing step, $\Gamma=0.5, \Lambda=0.25)$ : a) $\operatorname{Ra}=2 \times 10^{3}, \psi_{\max }$ $=0.53$; b) $\mathrm{Ra}=5 \times 10^{3}, \psi_{\max }=1.26$.
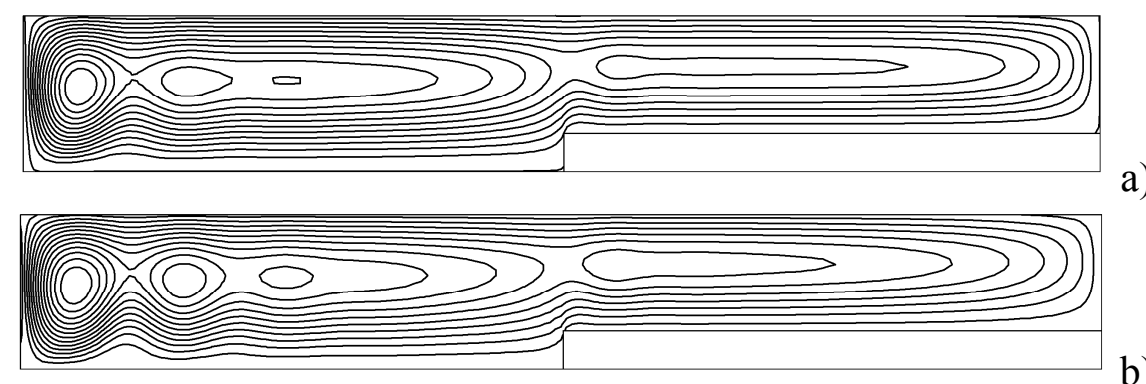

a)

b)

Figure 7: Streamlines $(\operatorname{Pr}=0.01, \mathrm{~A}=20$, Forward-facing step, $\Gamma=0.5, \Lambda=0.25)$ : a) $\operatorname{Ra}=2 \times 10^{3}, \psi_{\max }=$ 0.54 ; b) $\mathrm{Ra}=5 \times 10^{3}, \psi_{\max }=1.32$.

These differences, obviously, follow from the non-isotropy of the Hadley flow, which being a shear flow (directed from the hot side to the cold side at the free interface and in the reversed sense in proximity to the bottom), breaks the isotropy of the considered system with respect to the $\mathrm{x}$ coordinate. Though some limited differences can be noticed in the maximum value attained by the 
streamfunction, the most notable feature distinguishing the two patterns is the presence of an intense roll located in proximity to the section where the change in the cross-sectional area occurs (namely $\mathrm{x}=\mathrm{A} / 2$ ). Such a roll, clearly visible in Fig. 6, is completely absent in Fig. 7, where the flow in the region with reduced cross-sectional area $(\mathrm{x}>\mathrm{A} / 2)$ seems to be the "natural" continuation or extension of the flow established in the pre-step region. By contrast, the vortex located astride the step in Fig. 6 apparently breaks the almost parallel nature of the flow established in the region $\mathrm{x}$ $<\mathrm{A} / 2$, leading to the emergence of a multicellular pattern in the post step-region (the larger the Rayleigh number, the higher the number of rolls embedded in the overall convective system in the right part of the cavity).

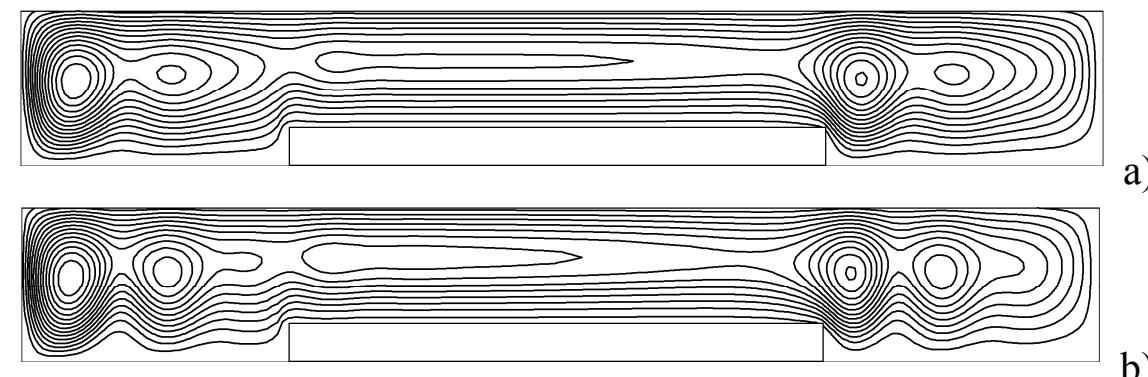

a)

b)

Figure 8: Streamlines $\left(\operatorname{Pr}=0.01, \mathrm{~A}=20\right.$, central obstruction, $\left.\Gamma_{1}=0.25, \Lambda=0.25\right)$ : a) $\operatorname{Ra}=2 \times 10^{3}, \psi_{\max }=$ 0.52 ; b) $\mathrm{Ra}=5 \times 10^{3}, \psi_{\max }=1.21$.

Such a roll is still a feature of the flow when the geometry with an obstruction located in the centre is considered (i.e., a reduction of the cross-sectional area for $\mathrm{A} / 4<\mathrm{x}<3 / 4 \mathrm{~A}$ ). The related results shown in Figure 8, however, reveal that its intensity is weakened with respect to the cavity with the backward-facing step. Moreover, in this case the flow in the region with reduced cross-sectional area displays again the quasi-parallel character already seen for the configuration with the forwardfacing step, which makes the resulting flow structure a kind of hybrid situation or "state" with respect to those illustrated in Fig. 6 and 7.

\subsection{Oscillatory States: Backward-facing step}

By increasing the Rayleigh number, the behaviours described in the preceding section are gradually taken over by more complex dynamics involving the onset of oscillatory disturbances (like those examined in Sect . 3.1 for the classical unobstructed rectangular cavity).

The numerical results, however, confirm that the presence of a topography in the cavity does still play a significant role.

As a first example, we consider the cavity with the backward-facing step, assuming the same value of the Rayleigh number used for the simulations shown in Fig. 5.

Direct comparison of the numerical results for this case (Fig. 9) with the canonical open cavity (no steps on the bottom) gives some immediate insights into the expected influence of geometrical effects. Indeed, the reader will easily realize that the constancy of frequency and amplitude of 
temperature signal is no longer a feature of the field (amplitude and frequency of the disturbance change significantly along the horizontal coordinate with respect to the case with straight bottom, Fig. 9a).

In particular, for $\Lambda=0.25$ the amplitude of oscillations increases in the region with reduced crosssectional area $(\mathrm{x}>\mathrm{A} / 2)$ whereas their main frequency undergoes a decrease.

Such changes are reflected in observable modifications of the flow structure and related spatiotemporal evolution (Fig. 9b). The resulting flow pattern can now be ideally split into two clearly distinguishable circulation systems:

A group of rolls is located in the post-step region $(x>A / 2)$. This region is characterized by a relatively stable pattern consisting of weakly oscillating rolls. Although these rolls undergo some weak growth and decay in time and/or a back and forth motion, they maintain relatively stable positions, being constrained to the right part of the cavity. In this regard, their behaviour is rather similar to that displayed by the classical hydrodynamic oscillatory disturbance in canonical rectangular cavities. Such a set of rolls is bounded from the left by the strong convective cells established astride the step as already discussed for the backward-facing step in Sect. 3.2 and by the hot wall from the right.

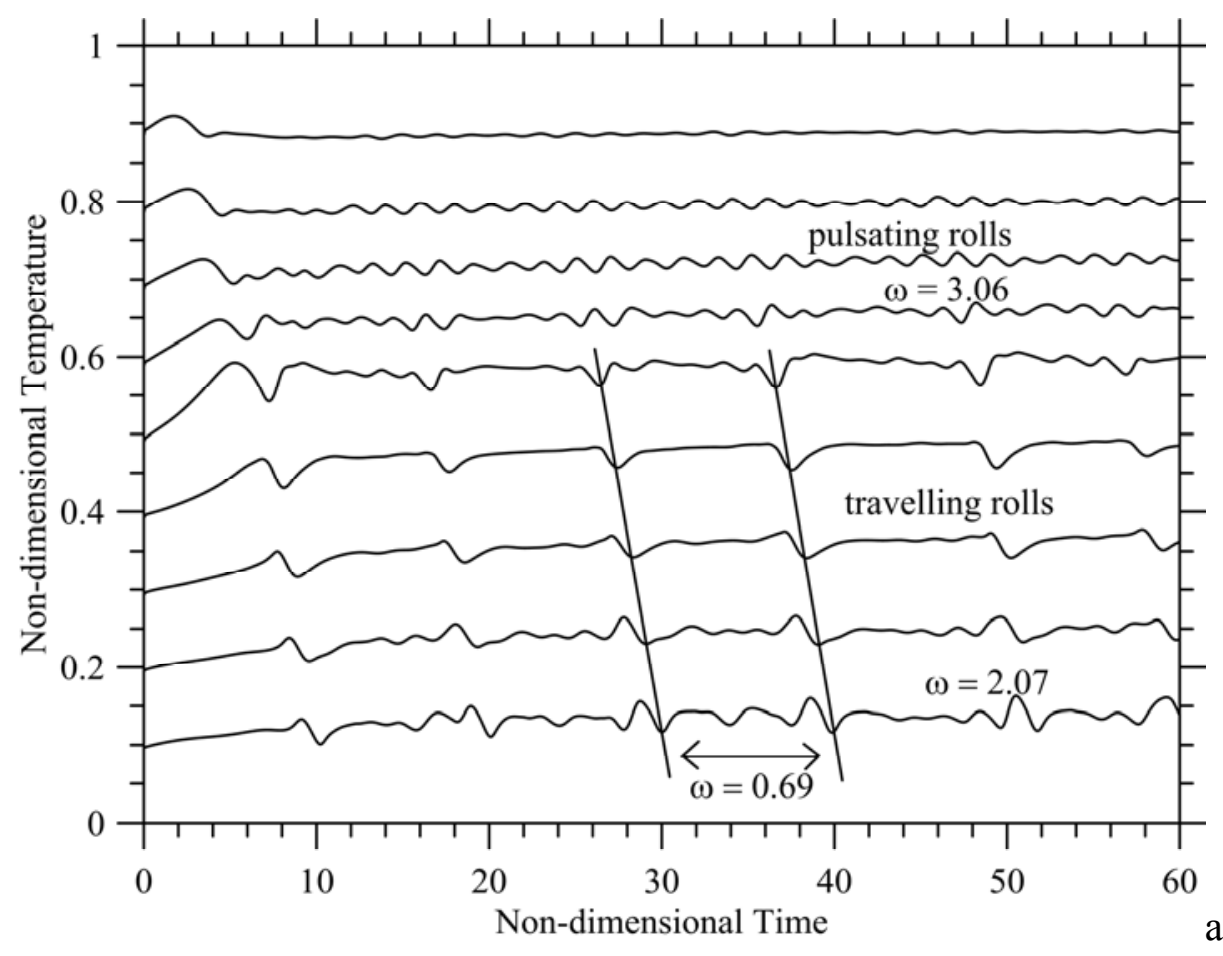




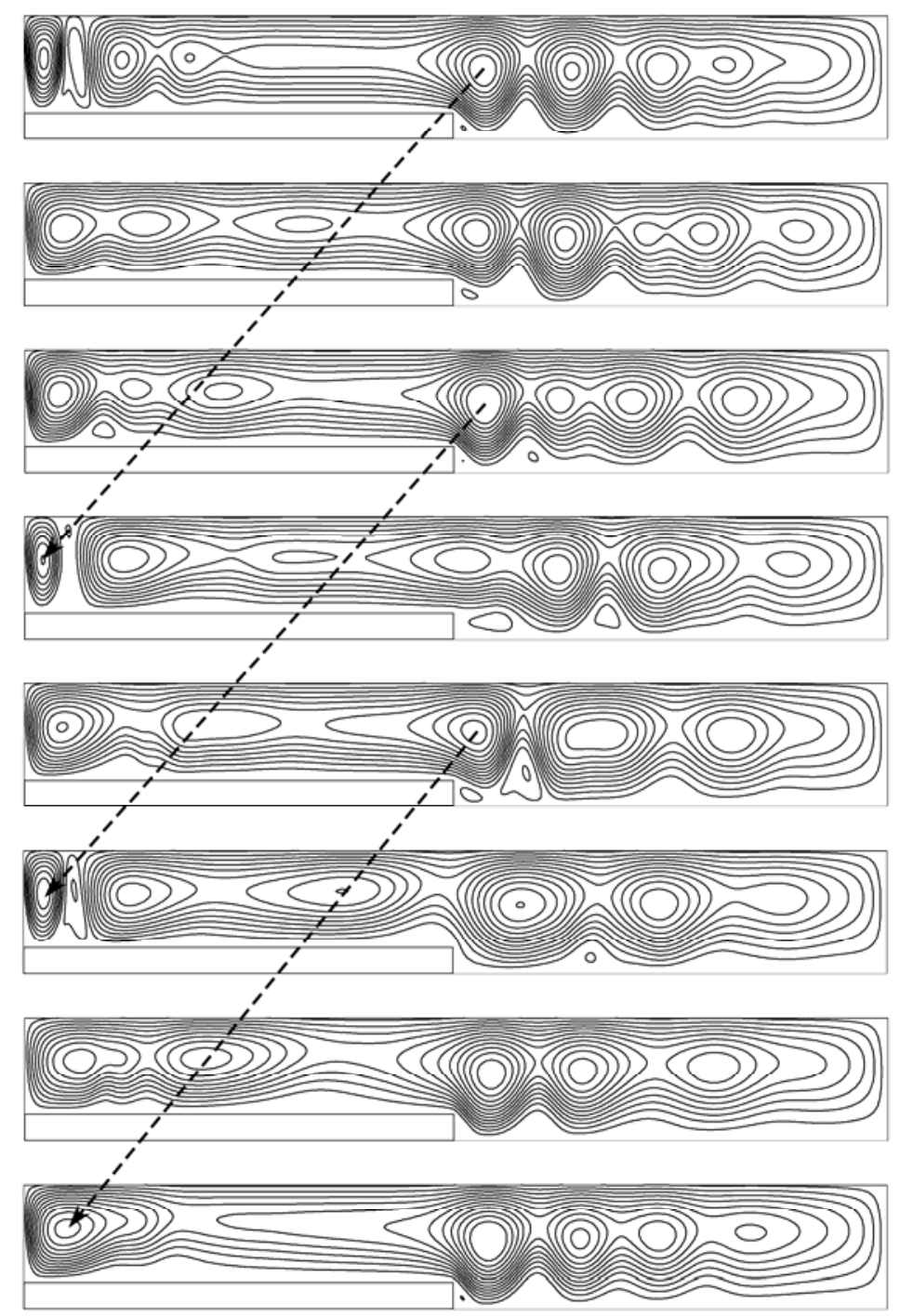

b)

Figure 9: $\left(\operatorname{Pr}=0.01, \mathrm{~A}=20\right.$, backward-facing step, $\left.\Gamma=0.5, \Lambda=0.25, \mathrm{Ra}=1.5 \times 10^{4}\right)$ : a) plot of signals provided by numerical probes; $b$ ) eight snapshots evenly distributed during one period of oscillation related to the angular frequency $\omega=0.69$.

If the oscillations are measured in points with the same vertical coordinates but at different horizontal positions, in this part of the cavity only two values of the horizontal phase shift are allowed $(\Delta \phi=0$ or $\Delta \phi=\pi)$; indeed, there is no phase shift between $\mathrm{T}_{6}$ and $\mathrm{T}_{8}$ and no phase shift between $T_{7}$ and $T_{9}$, but $T_{6}$ and $T_{8}$ measure values with a phase shift of $\pi$ with respect to $T_{7}$ and $T_{9}$ (Fig. 9a).

The dynamics, however, completely change when the part of the cavity located in the region of reduced cross-sectional area is considered $(\mathrm{x}<\mathrm{A} / 2)$. A convective cell spatially spreading periodically towards the cold side represents the prevailing convective system there (Fig. 9b). Such a phenomenon is bounded from the left (where the propagation velocity of the moving roll decays) by the cold wall and from the right (where such roll is being continuously created) by the aforementioned relatively strong roll steadily located astride the step. When the propagating roll 
"dies" in proximity to the cold side, a new roll is created at the other side of the region of reduced cross-sectional area thereby preserving the oscillatory nature of the phenomenon. Most remarkably, for $\mathrm{x}<\mathrm{A} / 2$, the values of the phases related to the signals provided by the thermocouples are not discrete. This is a completely new feature, rather unexpected given the "known" fluid-dynamic response of liquid metals to the application of vertical gravity and horizontal temperature gradients as discussed in the introduction. It can be seen that the main disturbance for $\mathrm{x}<\mathrm{A} / 2$ consists essentially of a perturbation traveling in the downstream direction, i.e. a "transverse wave" (a wave with its front perpendicular to the imposed temperature gradient).

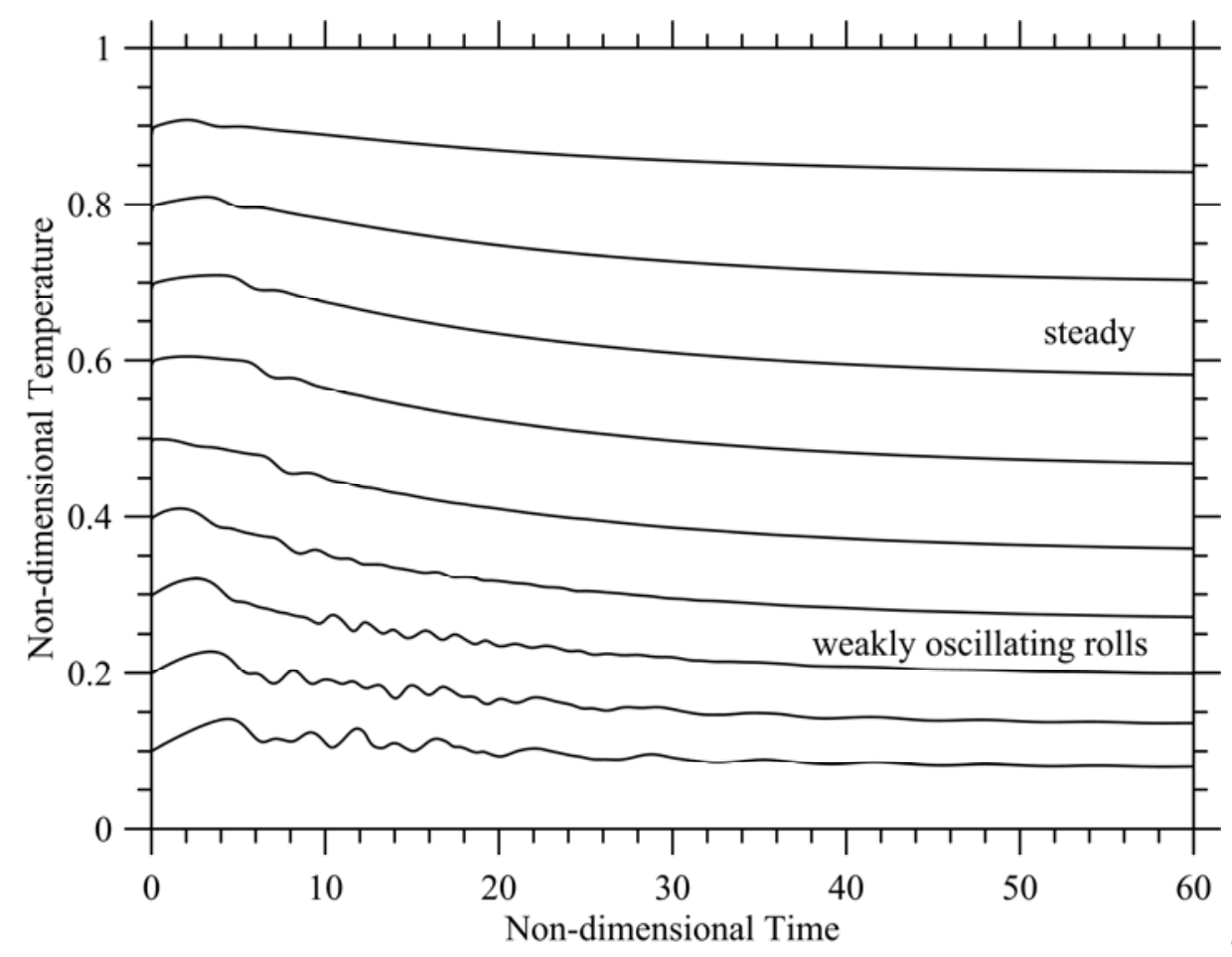

a)

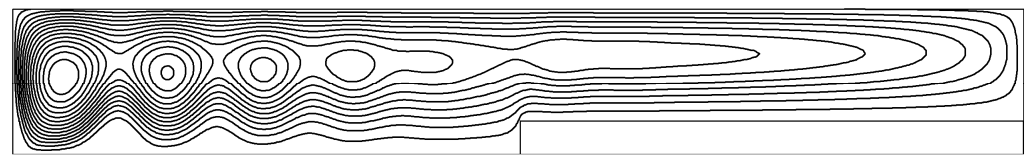

b)

Figure 10: $\left(\operatorname{Pr}=0.01, \mathrm{~A}=20\right.$, forward-facing step, $\left.\Gamma=0.5, \Lambda=0.25, \mathrm{Ra}=1.5 \times 10^{4}\right)$ : a) plot of signals provided by numerical probes; b) snapshot of streamlines distribution.

Waves travelling in the fluid are a well-known feature of the Hadley flow, but they are known to be excited only at values of the Prandtl number generally larger than that considered here (see the discussions at the beginning of Sect. 2.2). Moreover, such waves are known to travel in the spanwise direction, i.e. in a direction perpendicular to the basic flow and the applied temperature gradient (they are essentially a three-dimensional phenomenon as explained in the introduction). Their nature is different as well, as they are of a "hydrothermal nature", which means the instability does not depend solely on the presence of a shear stress. 
The present wave travelling in the streamwise direction (downstream) for $\operatorname{Pr}=0.01$ can be therefore regarded as a new phenomenon (possible analogies with other known mechanisms will be discussed in Sect. 4).

The dynamics are made even more interesting by the increased complexity visible in the frequency spectrum. The angular frequency related to the propagation of the wave to the left (detected by the thermocouples from $T_{1}$ to $T_{5}$ ) is clearly smaller than the typical angular frequency of the classical oscillatory disturbance excited in the right part of the cavity (as detected by the thermocouples from $\mathrm{T}_{6}$ to $\mathrm{T}_{9}$ ). The related values are $\omega_{\mathrm{wave}} \simeq 2.07$ and $\omega_{\text {dist }} \cong 3.06$, respectively.

Moreover, a "second" frequency $\omega_{\text {wave }} \cong 0.69$ can be identified in the typical temperature signals provided by the thermocouples in the region of reduced cross-sectional area. Being much smaller than the angular frequency with which the disturbance travels to the left, this second frequency seems to be related to a slow modulation of the aforementioned roll located astride the step (we will come back to this concept later).

\subsection{Oscillatory States: Forward-facing step}

As it could have been anticipated on the basis of the discussions elaborated in Sect. 3.2 for the low$\mathrm{Ra}$ regime, the problem is not invariant for reflections with respect to $\mathrm{x}=\mathrm{A} / 2$. For the forwardsfacing step case and the same conditions considered in Sect. $3.3\left(\Lambda=0.25\right.$ and $\left.R a=1.5 \times 10^{4}\right)$, the presence of the step leads to a complete stabilization of the flow in the right part of the cavity with the oscillatory disturbance of hydrodynamic nature surviving solely in the pre-step region (Fig. 10). This is witnessed by the signals provided by the thermocouples located along the horizontal direction, which display clearly observable oscillations only for $\mathrm{x} \leq 0.4 \mathrm{~A}$.

A decrease in the height of the step does not change significantly the dynamics, although, as shown in Fig. 11, the horizontal extension of the region where the oscillatory behaviour can survive increases (compare Fig. 11a and Fig. 10a) and an increased degree of complexity can be seen in the frequency spectrum (one or more additional frequencies seem to affect the signals provided by $\mathrm{T}_{1}$ and $\mathrm{T}_{2}$ in Fig. 11a). 

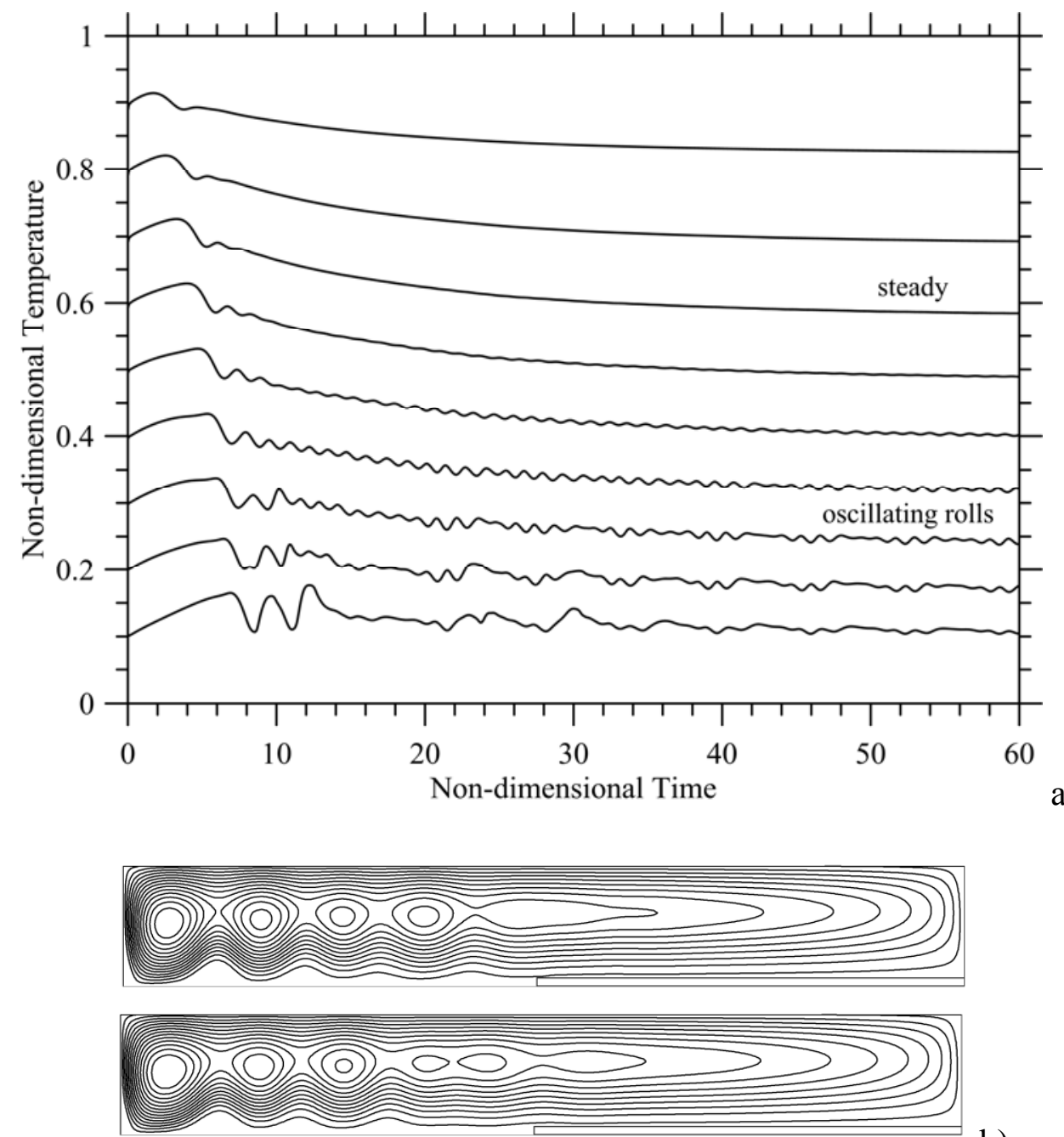

b)

Figure 11: $\left(\operatorname{Pr}=0.01, \mathrm{~A}=20\right.$, forward-facing step, $\left.\Gamma=0.5, \Lambda=0.05, \mathrm{Ra}=1.5 \times 10^{4}\right)$ : a) plot of signals provided by numerical probes; $b$ ) snapshots of streamlines distribution.

\subsection{Oscillatory States: Central Obstruction}

When the obstruction is located in the centre of the cavity, the scenario is qualitatively similar to that seen for the backward-facing step case. A series of two or three rolls embedded in the almost parallel flow established in the central region $(0.25 \mathrm{~A}<\mathrm{x}<0.75 \mathrm{~A}$, with fluid traveling from the hot side to the cold side at the free surface and in the opposite direction close to the bottom) become a persistent feature of the velocity field. By moving continuously along the horizontal direction, they create the illusion of an endless traveling train of vortices, i.e. a travelling "wave". Comparison of the different stages of evolution shown in Fig. 12b leads to the conclusion that the direction of propagation of such convective disturbances is still in the downstream direction. The related angular frequency, however, experiences a slight increase with respect to the value measured for the same value of the Rayleigh number and the same height of the obstacle in the backward-facing step case (see Fig. 9). Moreover, the dynamics are more regular, as witnessed by the lack of a clearly detectable second (smaller) frequency modulating the propagation of the rolls to the left. 

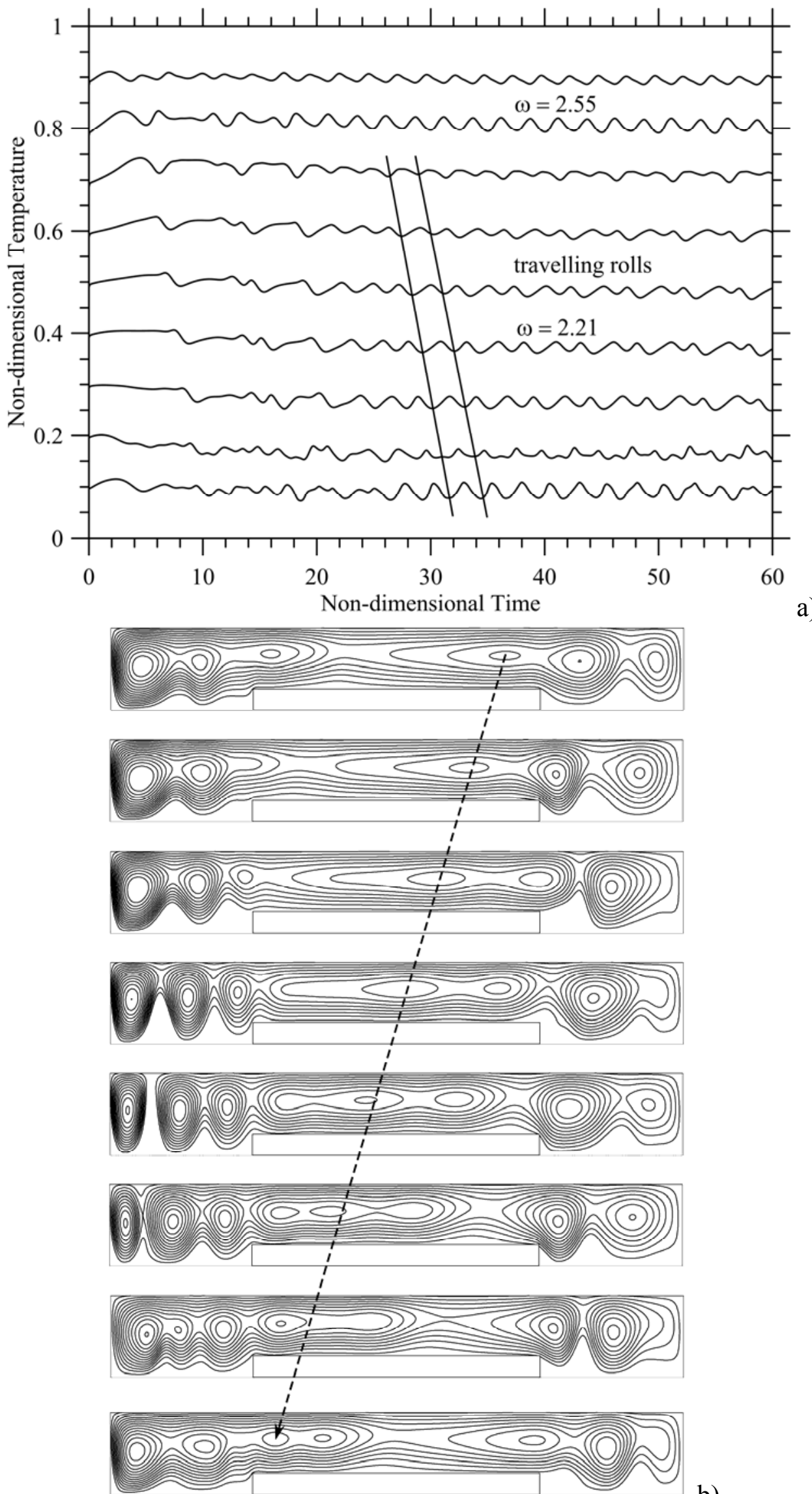

Figure 12: $\left(\operatorname{Pr}=0.01, A=20\right.$, central obstruction, $\left.\Gamma_{1}=0.25, \Lambda=0.25, \mathrm{Ra}=1.5 \times 10^{4}\right)$ : a) plot of signals provided by numerical probes; b) eight snapshots evenly distributed during one period of oscillation related to the angular frequency $\omega=2.21$. 
Additional meaningful information can be obtained by looking directly at the signals provided by the numerical probes. The typical signature of a disturbance travelling continuously along a given direction, indeed, is represented by the aforementioned continuous phase shift displayed by signals measured at different locations evenly spaced along the direction of propagation (Fig. 12a).

Such a continuous phase shift can be clearly distinguished in the signals measured by the thermocouples from $T_{1}$ and $T_{7}$. This observation indicates that the existence of the wave is not limited to the region of reduced cross-sectional area as it was in the backward-facing step case. Most notably, the propagation of the disturbance also extends to the pre-step region, i.e. $\mathrm{x}<0.25 \mathrm{~A}$. Another important distinguishing mark can be identified in the behaviour of the rolls at the other side of the cavity (where the travelling wave originates, i.e. the post-step region, $x>0.75 \mathrm{~A}$ ). The rolls there seem to actively participate to the overall mechanism supporting the propagation of the disturbance to the left. Indeed, a roll is periodically created at the hot side and moves to the left until it is squeezed and entrained in the region of reduced cross-sectional area (hereafter simply referred to as the "channel"). After being stretched along the horizontal direction, compressed along the vertical direction and "funnelled" into the channel, such a roll undergoes regular (at a constant velocity) migration, eventually forcing other rolls located in such a region to move to the left. When one of these rolls reaches the exit of the channel, it expands in the vertical direction recovering most of its initial size.

This effect, in turn, results in a new roll being periodically released in the pre-step region $(x<0.25 \mathrm{~A})$. Under the impact of such a roll coming from the right, the two pre-existing rolls located in such a part of the cavity tend to be initially compressed along the horizontal extension. As a natural consequence, an interesting behaviour is established locally, consisting of the temporal alternation of a local pattern with three distinct corotating rolls and an ensuing one with two rolls only. The shrinkage in the roll number results essentially from a vortex coalescence process (the two rolls located in proximity to the cold wall, which are initially compressed along the horizontal direction and stretched vertically, finally merge resulting in the formation of a new main vortex attached to the cold wall).

As a natural consequence of such complex scenario involving the delicate interplay of several mechanisms (rhythmic roll expansions and contractions along the vertical and horizontal directions at different locations, roll nucleation, deformation, transport and merging phenomena), the number of convective rolls present at any instant in the layer is not constant (it tends to vary from a minimum of five rolls to eight).

Most remarkably, not all of such phenomena occur with the same time scale, which provides a justification for the slightly different frequencies visible in Fig. 12a (though a wave travelling downstream becomes the dominant mode of convection over the entire layer, the irregularities/asymmetries of the considered geometry results in various weak modulation effects). A major difference with respect to the situation examined in Sect. 3.3 for the backward-facing step can be identified essentially in the different role played by the rolls pertaining to the post-step region, which now actively participate to the overall dynamics. Indeed, the slowly oscillating roll 
located astride the step is no longer a stable feature of the system (which, among other things might explain why the second relatively small frequency discussed in Sect. 3.3 is no longer present in the region featuring the propagation of the wave).

\subsection{Increasing the step height}

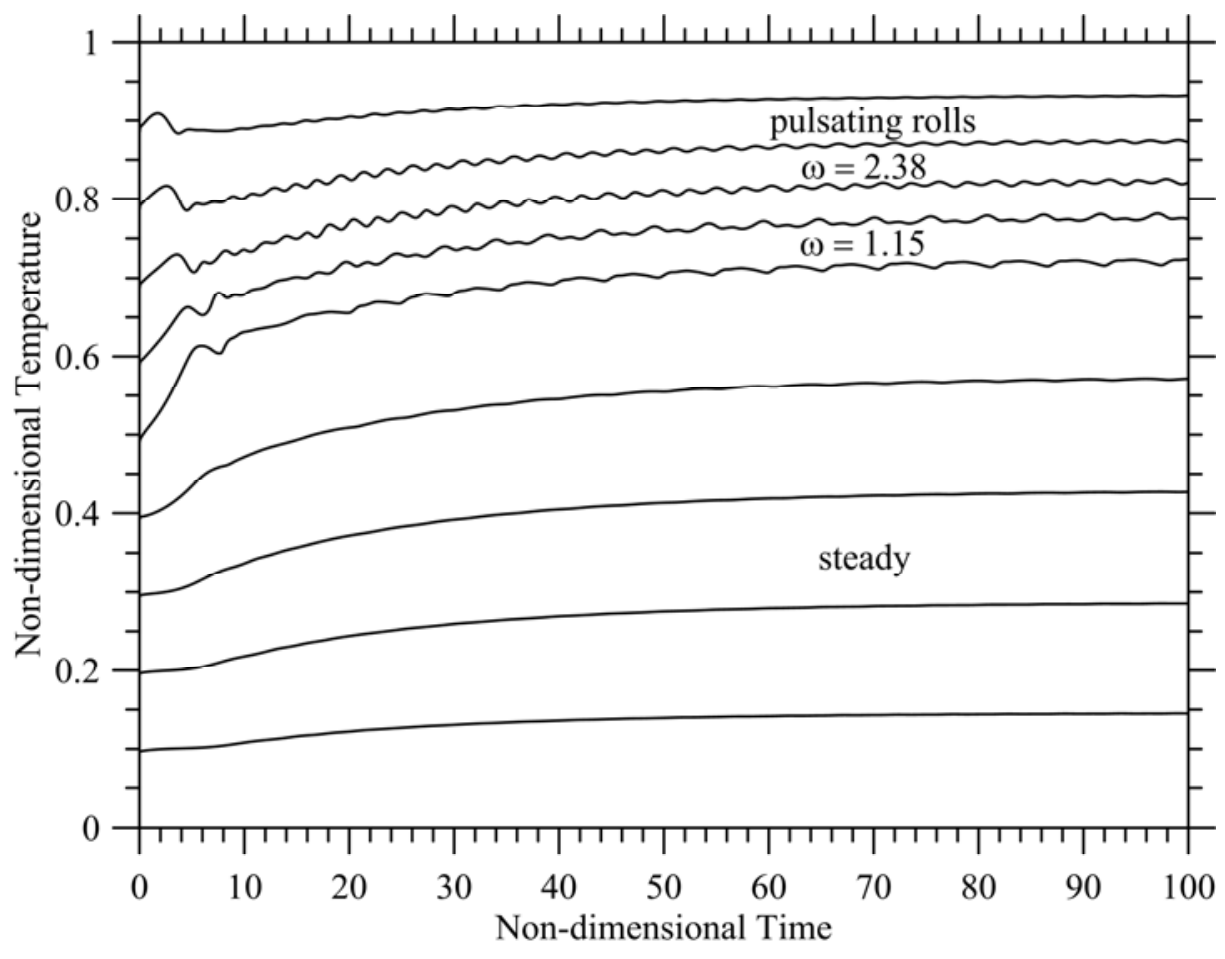

a)
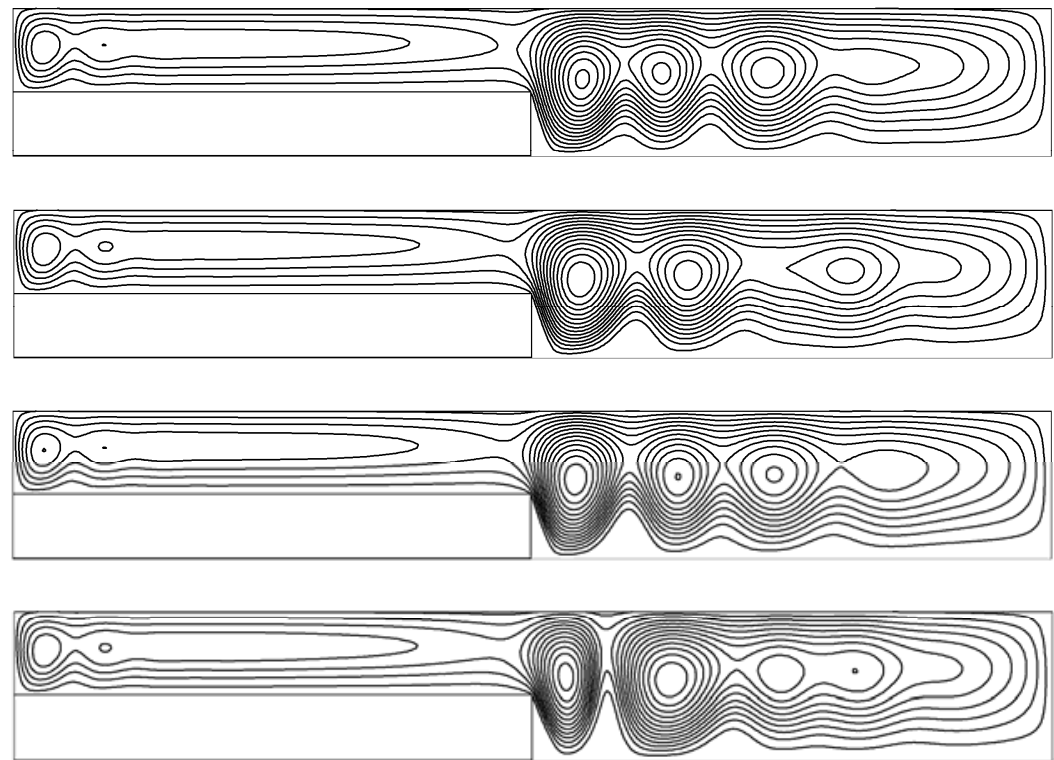

b)

Figure 13: $\left(\operatorname{Pr}=0.01, A=20\right.$, backward-facing step, $\left.\Gamma=0.5, \Lambda=0.45, \operatorname{Ra}=1.5 \times 10^{4}\right)$ : a) plot of signals provided by numerical probes; $b$ ) four snapshots evenly distributed during one period of oscillation related to the angular frequency $\omega=1.15$. 
This section is devoted to assessing the role played by an increase in the step height for the cases considered in Sects. 3.3 and 3.5 (such a study would make no sense for the forward-facing step for which we have already shown that the effect of the step height is limited to exerting a weak influence of the horizontal extension of the region displaying the oscillatory behaviour typical of the classical hydrodynamic disturbances).

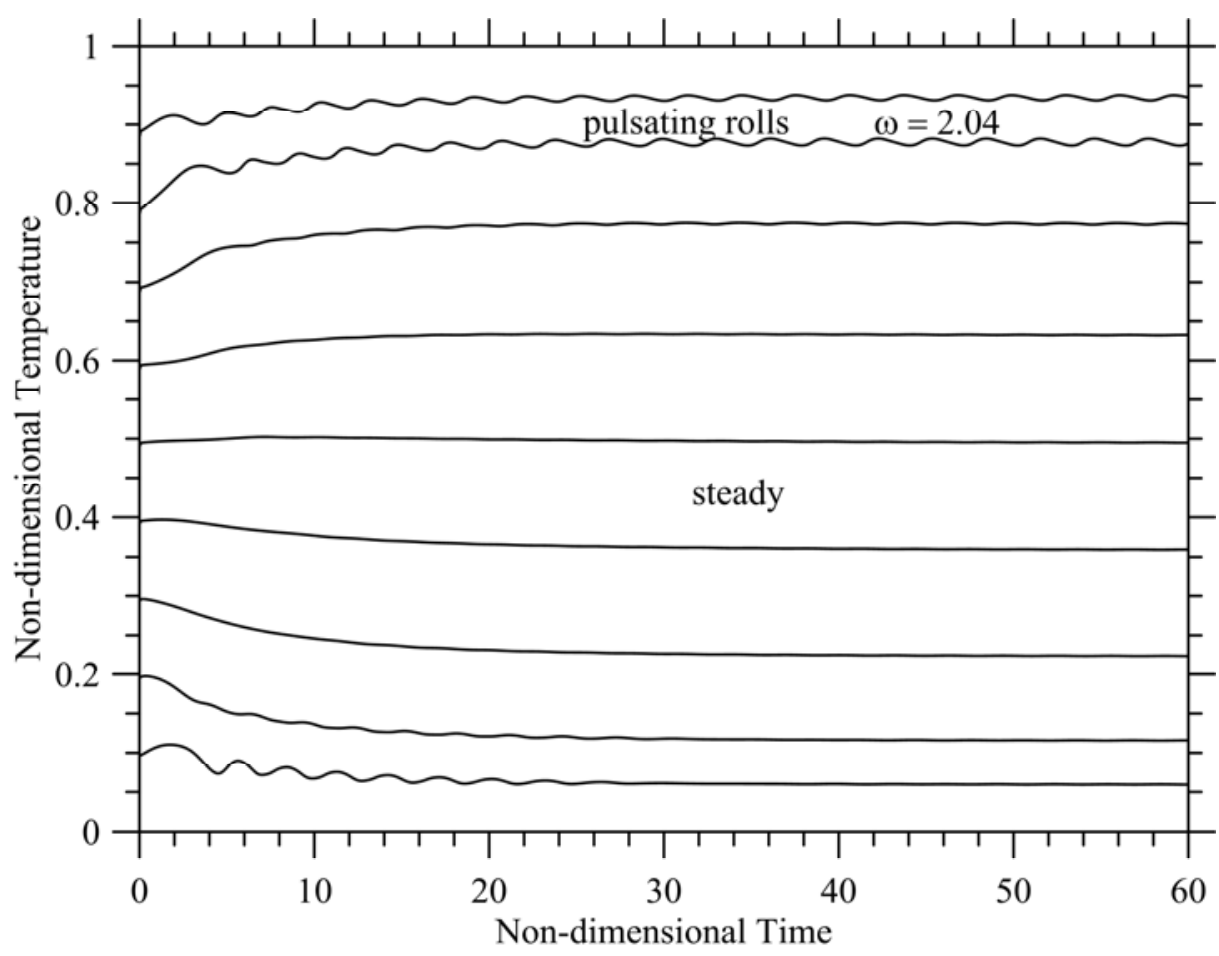

a)
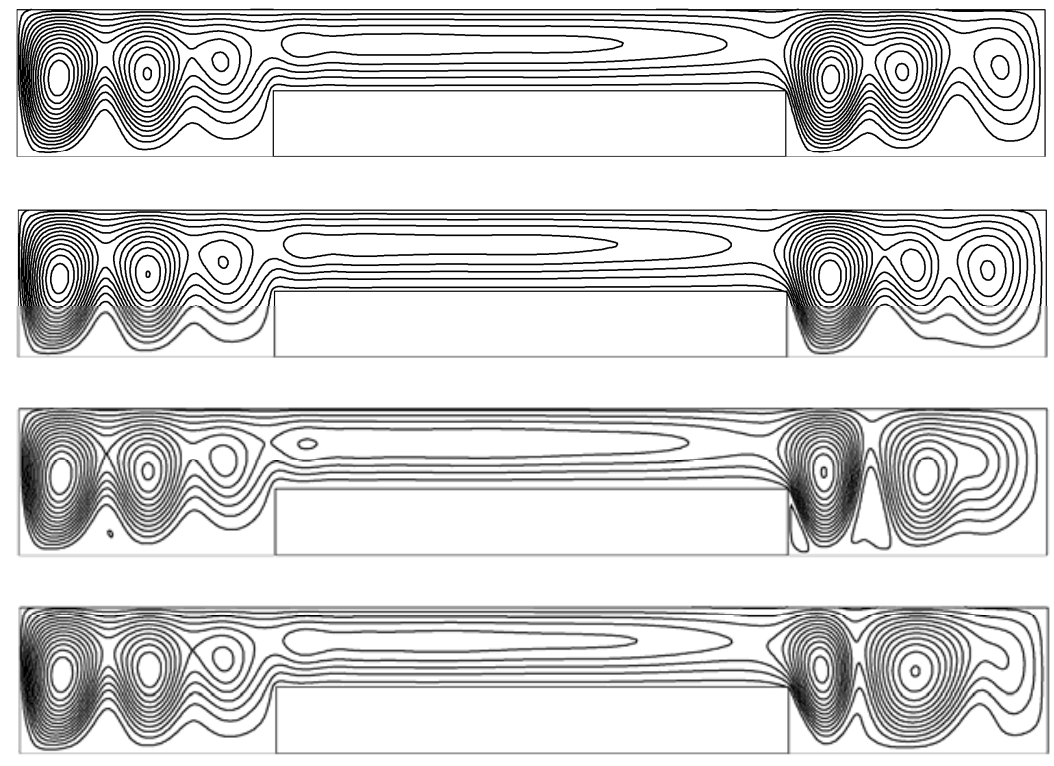

b)

Figure 14: $\left(\operatorname{Pr}=0.01, \mathrm{~A}=20\right.$, central obstruction, $\left.\Gamma_{1}=0.25, \Lambda=0.45, \mathrm{Ra}=1.5 \times 10^{4}\right)$ : a) plot of signals provided by numerical probes; a) four snapshots evenly distributed during one period of oscillation related to the angular frequency $\omega=2.04$. 
Figures 13 and 14 essentially show that an increase in $\Lambda(\Lambda=0.45)$ for the same value of the Rayleigh number considered before $\left(\mathrm{Ra}=1.5 \times 10^{4}\right)$ leads to an almost complete suppression of the travelling disturbance for both cases of backward-facing step and central obstruction. This, in turn, results in the recovery of conditions of almost perfect parallel flow in the region of reduced crosssectional area and in the ensuing decrease of the average number of rolls present in the cavity at any instant (rolls are located in the pre-step and/or post-step regions only, where the oscillatory behaviour consists essentially of signals featuring discrete phase shifts, like those that should be expected for the classical hydrodynamic disturbance).

The backward-facing step case deserves some additional attention as it might provide some useful insights into the complexity of the spectrum of frequencies seen in Fig. 9. Indeed, it shows that when the flow is forced to interact with an obstruction located in the cavity, but no traveling wave is produced in the region of reduced cross-sectional area, the frequency in the post-step region tends to behave essentially as a decreasing function of the distance from the step (see Fig. 15). In particular, the oscillation frequency of the roll steadily located astride the step is almost the half of the frequency of the other rolls lying in the post-step region, which may provide a justification for the existence of the third relatively small frequency discussed in Sect. 3.3.

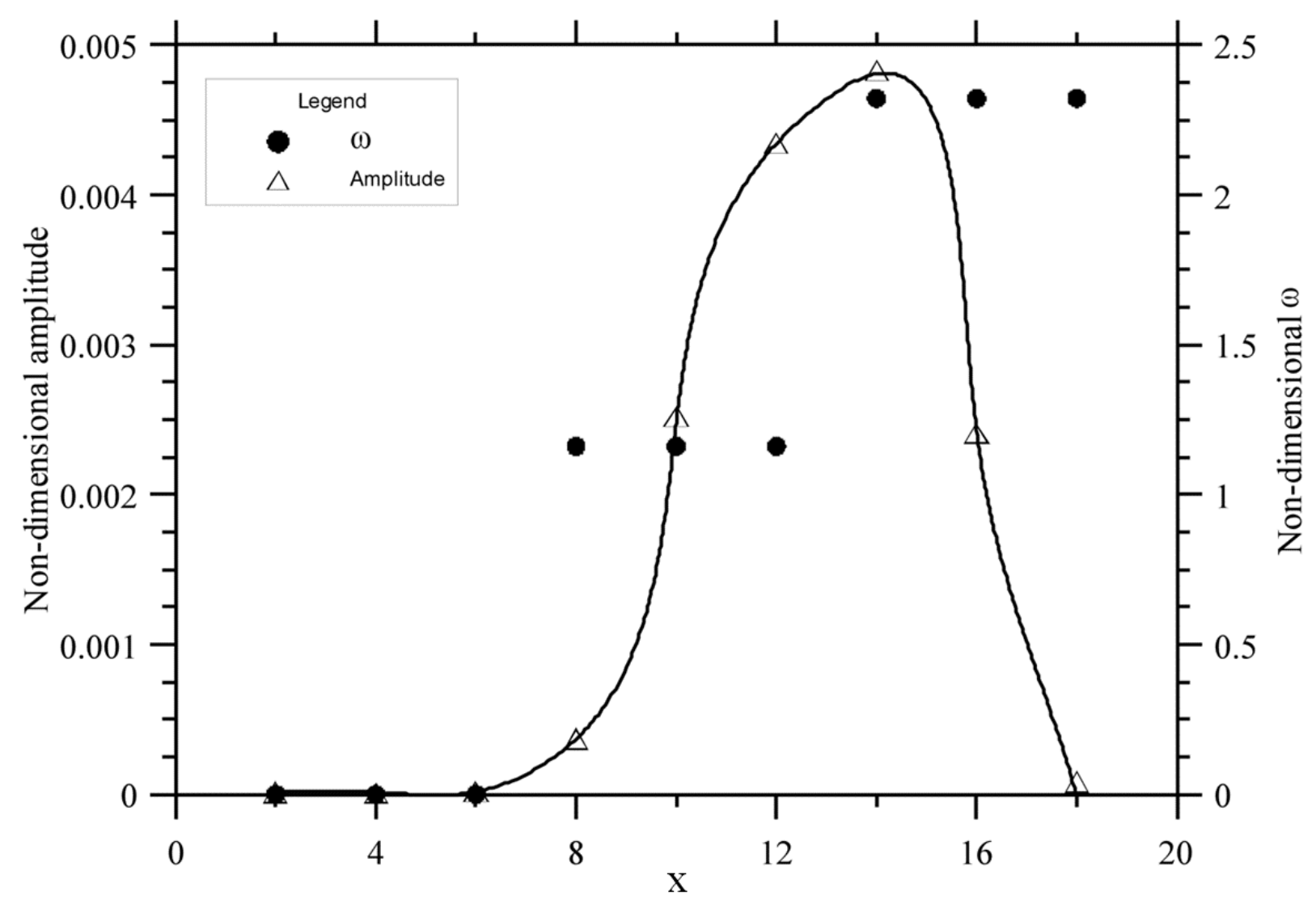

Figure 15: $\left(\operatorname{Pr}=0.01, A=20\right.$, backward-facing step, $\left.\Gamma=0.5, \Lambda=0.45, \mathrm{Ra}=1.5 \times 10^{4}\right)$ Nondimensional oscillations amplitude and frequency along the horizontal extension of the cavity. 


\section{Wavelike disturbances and vortex detachment}

In this section we further discuss the properties and the possible origin of the "transverse wave" revealed by the present study as a heretofore unseen characteristic of the Hadley flow of liquid metal for the cases with the central obstruction and the backward-facing step. Indeed, vortex breaking and/or detachment phenomena around topographic features and the evolution of waves on vorticity fronts are not new in fluid-dynamics (which makes an attempt to interpret such phenomena in the light of existing knowledge on the subject a worthy effort). Similar mechanisms can be found in disparate fields ranging from aeronautics and related aerodynamics problems to a number of industrial applications in the field of mechanical engineering (transition in parallel flows such as plane channels and pipes with blockage effects, Gresho et al. [38]), typical problems of biomechanics (such as the flow of blood through an obstructed arterial passage, Griffith et al. [39]) and even the dynamics of large-scale flow in the atmosphere or in the ocean in the presence of a topographic forcing (e.g., coastal mesoscale phenomena, Viera and Grimshaw [40]).

For all such cases it is known that a topography can be potentially responsible for a number of phenomena such as the onset and growth of small amplitude wavelike disturbances, the formation of filaments, winding of filaments (or of a vortex) around a topographic feature and the periodic (or aperiodic) release of eddies.

Though many of these effects are known to be intrinsically three-dimensional and for most of them and exhaustive explanation has not been elaborated yet (Griffith et al. [39]), for the specific case of two-dimensional flows such as those considered in the present work, some relevant information can be found in Boghosian and Cassel $[41,42]$. These authors categorized potential mechanisms thought to be responsible for the genesis of the vortex splitting phenomenon (and ensuing periodic shedding of eddies) in both internal and external flows as follows:

A first mechanism is represented by the classical inviscid Kelvin-Helmholtz instability in a free shear layer leading the fluid to "roll up" into a chain of vortices that subsequently detach from the layer. Vortices can be also produced via a process generally referred to as "pinch-off", which occurs when a sufficient amount of vorticity is accumulated in a localized region (this pinch-off process relies on the so-called Kelvin-Benjamin variational principle, Gharib et al. [43]). Other explanations for this phenomenon also include topological flow changes inside a recirculation region and pressure-gradient driven mechanisms [41]. An analysis of the most probable cause-and-effect relationships supporting the vortex splitting process under the constraint of two-dimensional flow, in particular, is due to the aforementioned Boghosian and Cassel [41,42], who found this mechanism (termed the "vortex shedding mechanism" (VSM)) to be a kind of (shear driven) Kelvin-Helmholtz instability dependent on two fundamental requirements only, namely, the existence of a location where the momentum is negligible and a force having a positive divergence. Most interestingly, these authors identified multiple sources potentially supporting the emergence of such a force, such as inherent nonlinearities in the Navier-Stokes equations and especially disturbances in the form of spatially growing waves. Among other things, this interpretation seems 
to be in line with existing stability analyses on the subject such as those by Kaiktsis et al. [44] and Wee et al. [45].

In particular, as reported in Wee et al. [45] and Boghosian and Cassel [41], a justification for the relevance of this interpretation to most of the observed phenomena in the literature (for both constrained or topography-disturbed internal and external flows), would be represented by the more or less universal behaviour taken by the nondimensional frequency of the travelling eddies when expressed in terms of the so-called Strouhal number:

$S t=\frac{f d}{U}=O\left(10^{-1}\right)$

where $f$ is the frequency of oscillations $(\omega / 2 \pi), d$ the thickness of the layer and $U_{\operatorname{dim}}$ the dimensional average velocity. The Reynolds number $\left(\operatorname{Re}=U_{\operatorname{dim}} \mathrm{d} / \mathrm{v}\right)$ leading to the onset of instability was found to be $\mathrm{O}\left(10^{3}\right)$ in these analyses.

Evaluation of these numbers for the conditions corresponding to Fig. 9 or Fig. 12 (the value of $U$ being extracted directly from the numerical simulations and St computed as $\omega \mathrm{d} / 2 \pi \mathrm{U}$ ) can be given as follows:

\begin{tabular}{ccc} 
Case & $R e$ & $S t$ \\
\hline backward-facing step,$\Lambda=0.25$ & $\cong 700$ & $\cong 0.053$ \\
central obstruction, $\Lambda=0.25$ & $\cong 700$ & $\cong 0.058$
\end{tabular}

Table IV: Reynolds and Strouhal numbers for $\mathrm{Ra}=1.5 \times 10^{4}$ and different conditions.

The present values of the Reynolds and Strohual numbers would indicate a possible kinship of the present mechanism with the general class of instabilities identified by Wee et al. [45] and Boghosian and Cassel [41,42]. As in our case the flow over the step includes both a direct and a return flow, whereas in the above studies the flow was assumed to be unidirectional, such interpretation, however, is not conclusive and would require some additional work in the future (being specifically concentrated on performing a linear stability analysis for the conditions considered in the present work).

As a concluding remark for this section, we discuss shortly our findings about the "reduced" number of cells in both Figs. 9 and 12 present above the steps (where the vortex shedding mechanism is established) with respect to those that would be supported by a cavity with "equivalent" (local depth to step horizontal extension ratio) aspect ratio. From a purely theoretical standpoint, it is known that, in the classical case of rectangular cell with no topography on the bottom, the spacing of the cells pertaining to the $2 \mathrm{D}$ hydrodynamic mode of liquid metals can be assumed to depend on two factors only. First is the fact that an integral number of cells must be accommodated into a cavity of finite length and the cell spacing must satisfy this constraint. The 
second factor is the magnitude of the departure from the onset of instability. The present results show that such criteria (valid for the classical rectangular cavity, Gelfgat et al. [31]) cannot be applied to the region of fluid located above the step (assuming this region to behave as it was an isolated cavity) because the number of rolls present there is significantly smaller. This discrepancy should be ascribed to the dynamic coupling established among the fluid behaviour in the different regions (pre-, above or post-step) of the cavity and the specific (travelling) behaviour displayed by the disturbances developing above the step. Such differences further reinforce the idea that the travelling wave in the step region has quite a different physical origin with respect to the classical pulsating rolls traditionally observed as the primary mechanism of instability of the Hadley flow in liquid metals.

\section{Surface tension-driven effects}

Such a case deserves attention as well. There is indeed quite a long tradition of studies in the literature expressly devoted to address the potential competition of thermocapillary and thermogravitational effects in determining the oscillatory response of layers of liquid metals or organic fluids to the application of horizontal temperature gradients (Okano et al. [46,47]; Shevtsova et al. [48]). From a historical standpoint, for configurations with adiabatic boundaries, such as those considered here, interesting information was initially provided by Laure and Roux [10]. Notably, for such thermal boundary conditions and relatively small values of the Prandtl number $(\mathrm{Pr}=0.02)$, these investigators found surface Marangoni stresses to exert a profound stabilizing effect on the $2 \mathrm{D}$ hydrodynamic mode of gravitational nature already for $\mathrm{W} \geq 0.02$ (their analysis concentrated on the range $0 \leq \mathrm{W} \leq 0.04$ ).

Yet through 2D numerical simulation, Ben Hadid and Roux [49,50] and Mundrane and Zebib [51] confirmed the stabilizing role played on the 2D hydrodynamic mode by Marangoni effects for $\operatorname{Pr}<<1$ in the range $0<\mathrm{W} \leq 0.04$ (in qualitative agreement with the earlier predictions yielded by Laure and Roux [10] in the framework of a linear stability analysis for the infinite layer).

This mode is completely suppressed when the strength of Marangoni convection is sufficiently increased in comparison to the thermogravitational counterpart $\left(\mathrm{W} \geq \mathrm{O}\left(10^{-1}\right)\right)$. As an example, for $\operatorname{Pr}=0.01$ Gershuni et al. [52] found $\mathrm{Ra}_{\mathrm{cr}} \rightarrow \infty$ for $\mathrm{W} \cong 0.0675$ (the reader being also referred to Henry et al. [53]).

Following such earlier studies valid for the classical unobstructed (rectangular) cavity or the infinite layer, in this section we finally examine the effect exerted by relatively weak surface tension effects on the travelling wave extensively discussed in the preceding sections, which of the present work represents the most interesting (heretofore unseen) finding. In particular, assuming the same value of the Rayleigh number and nondimensional step height considered in Sects. 3.3 and 3.5 $\left(\mathrm{Ra}=1.5 \times 10^{4}, \Lambda=0.25\right)$, we focus on two values of the parameter $\mathrm{W}$, i.e., $\mathrm{W} \cong 0.0067$ and $\mathrm{W} \cong 0.067$ corresponding to $\mathrm{Ma}=100$ and $\mathrm{Ma}=1000$, respectively. 

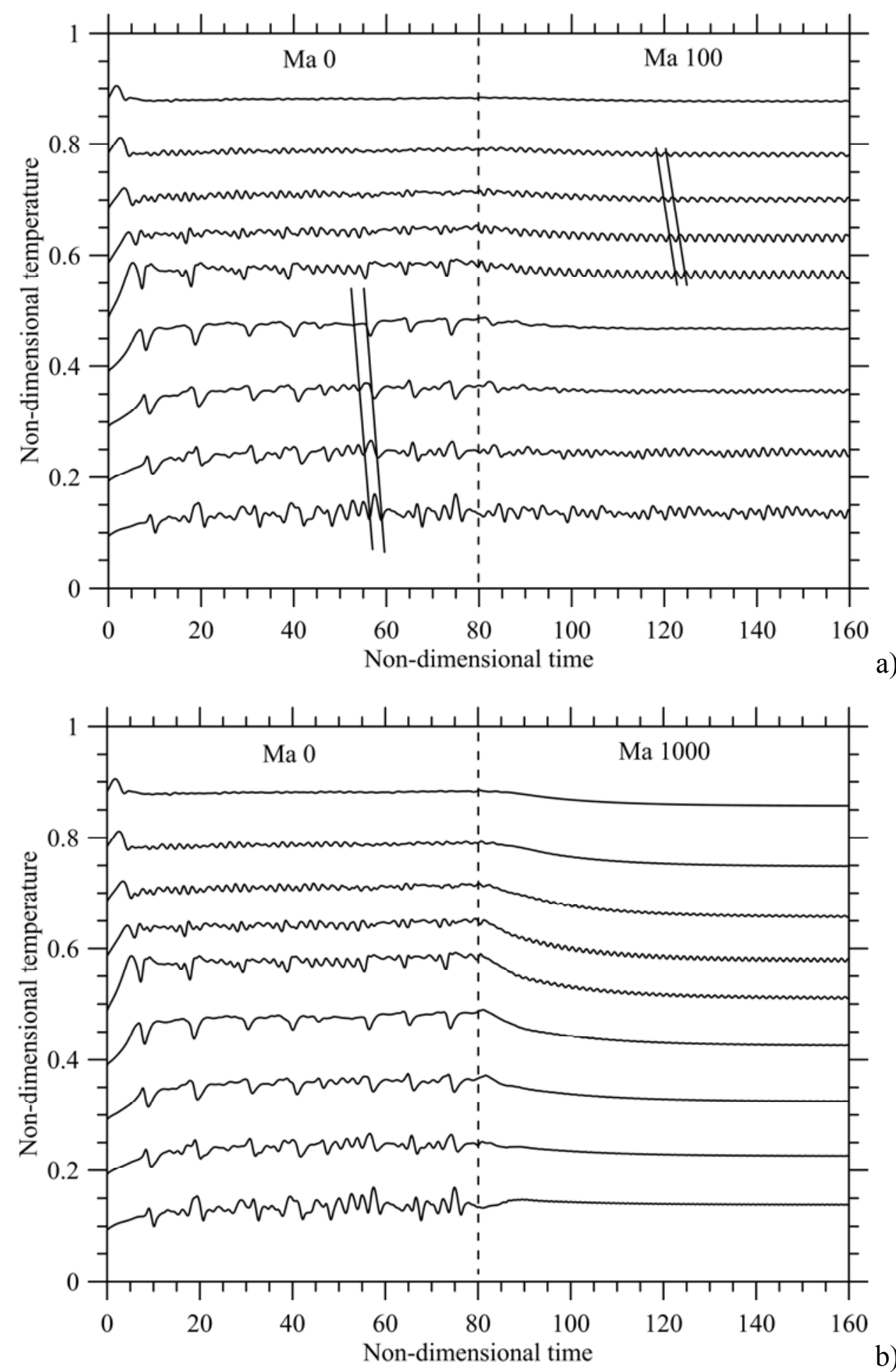

Figure 16: $\left(\operatorname{Pr}=0.01, \mathrm{~A}=20\right.$, backward-facing step, $\left.\Gamma=0.5, \Lambda=0.25, \mathrm{Ra}=1.5 \times 10^{4}\right)$ : a) plot of signals provided by numerical probes for $\mathrm{Ma}=100$; b) of signals provided by numerical probes for $\mathrm{Ma}=1000$.

The related results summarized in Figures 16 and 17, confirm that the influence of the Marangoni effect can be still considered stabilizing as it leads to a complete suppression of the travelling disturbance for $\mathrm{W} \cong 0.067\left(\mathrm{Ma}=10^{3}\right)$. It could be stated that the downstream travelling wave is even 
more sensitive to the "contamination" by Marangoni flow than the classical hydrodynamic disturbance, as witnessed by the strong mitigation of oscillations observable for $\mathrm{W} \cong 0.0067$ $(\mathrm{Ma}=100)$ in the central region as compared to the almost undisturbed oscillatory behaviour surviving for $\mathrm{x}>0.75 \mathrm{~A}$ (Fig. 17a).
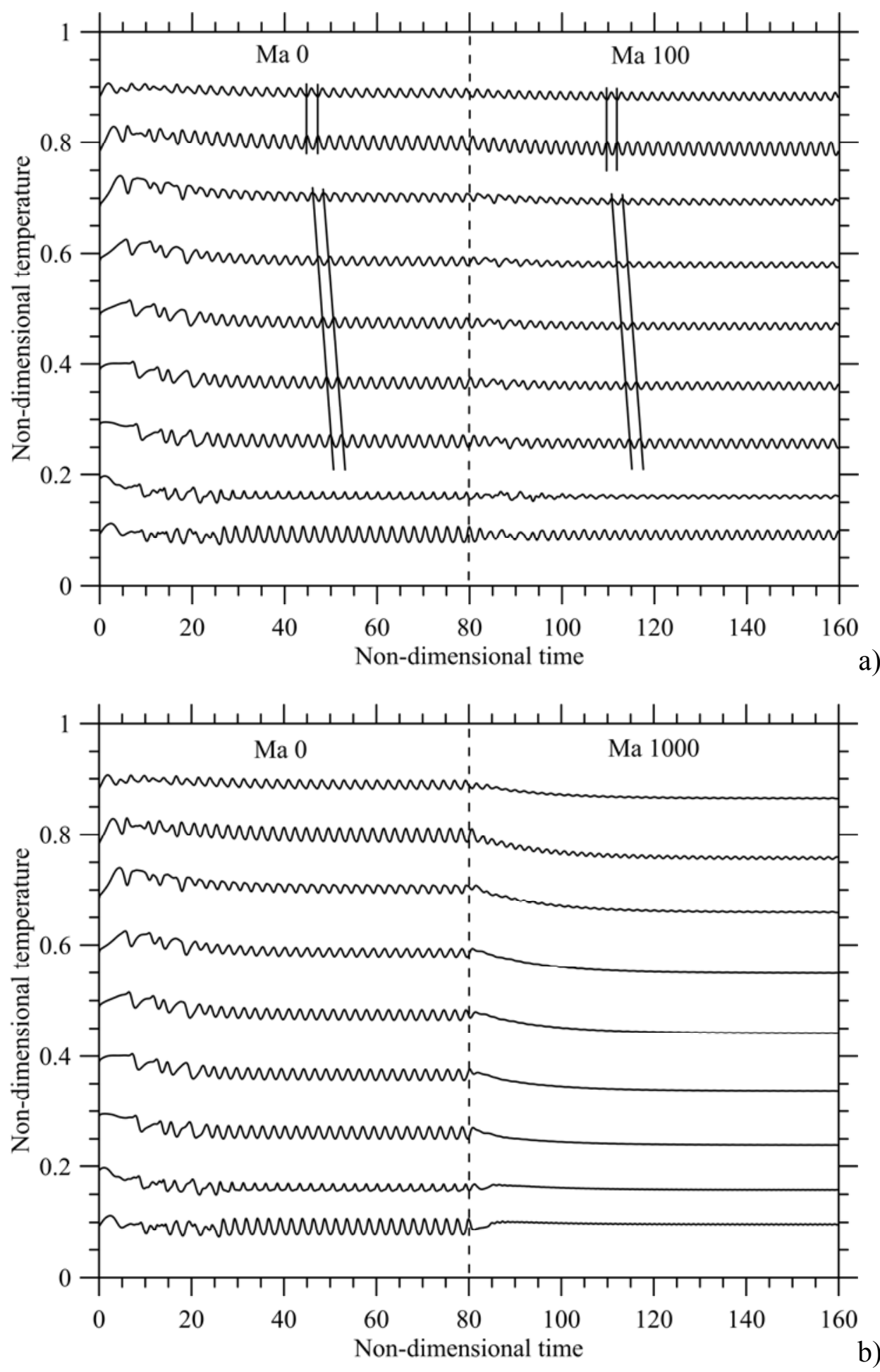

Figure 17: $\left(\operatorname{Pr}=0.01, \mathrm{~A}=20\right.$, central obstruction, $\left.\Gamma_{1}=0.25, \Lambda=0.25, \mathrm{Ra}=1.5 \times 10^{4}\right)$ : a) plot of signals provided by numerical probes for $\mathrm{Ma}=100 ; \mathrm{b}$ ) of signals provided by numerical probes for $\mathrm{Ma}=1000$. 


\section{Conclusions}

So far, research activity dealing with the typical instabilities of liquid metals in layers and horizontally extended cavities of finite extent, assumed as models of effective crystal growth processes, has been devoted especially to geometries displaying relatively simple (regular) shapes and symmetries with respect to the vertical and horizontal directions.

In general, because these well-defined geometric shapes render mathematical analysis and simulations simpler, they have enjoyed a widespread use in the definition and ensuing analysis of this subject (these models have indeed proved to retain the "essence" of the problem). Although there is no doubt that these simplified formulations have provided a great amount of valuable information over the years, however, circumstances exist for which a detailed and quantitative comparison with real situations is not possible. Given the absence of numerical studies addressing the interplay of oscillatory hydrodynamic modes with sudden variations of the available crosssectional area of the container for low-Pr liquids, in particular, we have focused expressly on such cases.

Following earlier efforts in the literature, in particular, we have considered the "typical" substances which have already enjoyed a widespread consideration for such a kind of studies, namely, silicon melt with $\operatorname{Pr}=0.01$, providing specific numerical examples expressly conceived and presented to provide inputs for an increased understanding of the underlying fluid flow mechanisms.

The most interesting outcomes of such a study can be summarized as follows.

Depending on the type of considered geometry change (forward-facing, backward-facing or both steps) the rolls associated with the hydrodynamic disturbance, which in general display a relatively simple oscillatory behaviour (a limited back and forth motion with no preferred direction of migration, in other words, a kind of "pulsating" mechanism, typically associated with limited changes in their intensity or strength), self-organize giving rise to a disturbance continuously spreading in the downstream direction, i.e. a wave. Sometimes such a wave exists as the dominant mode of convection, i.e. it occupies more or less the entire layer, while in other circumstances its presence is confined to a specific sub-region. In the latter case the coexistence of different oscillatory patterning behaviours (displaying different amplitudes and eventually frequencies) becomes an interesting feature of the flow.

The intrinsic properties of such a wave (its direction of propagation parallel to the imposed temperature gradient with travelling rolls having axes perpendicular to the basic flow) support the conclusion that such a phenomenon is still of a hydrodynamic kind, as the so-called "helical waves" of hydrothermal nature, which are also known to potentially affect gravitationally unstable liquid metals, generally travel in the spanwise direction, i.e. perpendicularly to the basic flow (being oscillatory and three-dimensional at the same time).

This new phenomenon can be completely suppressed or mitigated by an increase in the height of the step and/or by the presence of surface-tension driven effects (the so-called Marangoni flow), 
provided such a flow is not too strong (in which case the problem would be made more complex by the potential excitation of another kind of travelling waves which are specific to this kind of flow; see, e.g., Parmentier et al. [54]).

Future work shall be devoted to assess the influence exerted by such changes in the geometry boundary on the typical three-dimensional instabilities which occur for slightly larger values of the Prandtl number, namely the aforementioned helical or hydrothermal modes.

\section{Acknowledgements}

The author would like to thank Marco Camarotto for running the code and preparing most of figures reported in this work. He also participated actively in the discussions leading to the identification of the various mechanisms at play in such dynamics.

\section{References}

[1] Lappa M., (2005), Thermal convection and related instabilities in models of crystal growth from the melt on earth and in microgravity: Past history and current status, Cryst. Res. Technol., 40(6): 531-549.

[2] Lappa M., Thermal Convection: Patterns, Evolution and Stability (John Wiley \& Sons, Chichester, England, 2009).

[3] Dupret F. and Van der Bogaert N., (1994) Modelling Bridgman and Czochralski growth, in Handbook of Crystal Growth (ed. D. T. J. Hurle) 2: 877-1010. North-Holland, Amsterdam.

[4] Monberg E., (1994), Bridgman and related growth techniques. In Handbook of Crystal Growth (ed. D. T. J. Hurle), 2: 53-97. North-Holland, Amsterdam.

[5] Hadley G., (1735), Concerning the cause of the general trade winds, Phil. Trans. Roy. Soc. Lond., 29: 58-62.

[6] Hurle D.T.J., (1966), Temperature oscillations in molten metals and their relationship to growth striae in melt-grown crystals, Phil. Mag., 13: 305-310.

[7] Hart J.E., (1972), Stability of thin non-rotating Hadley circulations,. J. Atmos. Sci., 29: 687-697.

[8] Hart J.E., (1983), A note on the stability of low-Prandtl-number Hadley circulations, J. Fluid Mech., 132: 271-281.

[9] Gill A. E., (1974), A theory of thermal oscillations in liquid metals, J. Fluid Mech. 64 (3): 577 588.

[10] Laure P. and Roux B., (1989), Linear and non linear study of the Hadley circulation in the case of infinite cavity, J. Cryst. Growth 97(1): 226-234.

[11] Wang T.-M. and Korpela S.A., (1989), Longitudinal Rolls in a Shallow Cavity Heated from a Side, Phys. Fluids A, 32: 947-953.

[12] Okada K. and Ozoe H., (1993), The Effect of Aspect ratio on The critical Grashof number for Oscillatory Natural Convection of Zero Prandtl Number Fluid: Numerical Approach, J. Cryst. Growth, 126: 330-334. 
[13] Gelfgat A.Yu., Bar-Yoseph P.Z. and Yarin A.L., (1997), On Oscillatory instability of Convective Flows at Low Prandtl Number, Journal of Fluids Engineering, 119: 823-830.

[14] Ben Hadid H., Henry D., Kaddeche S., (1997), Numerical study of convection in the horizontal Bridgman configuration under the action of a constant magnetic field. Part 1. Two-dimensional flow, J. Fluid Mech., 333: 23-56.

[15] Kaddeche S., Henry D., Putelat T., Ben Hadid H., (2002), Instabilities in liquid metals controlled by constant magnetic field, Part I : vertical magnetic field, J. Cryst. Growth, 242: 491500.

[16] Kaddeche S., Ben Hadid H., Putelat T., Henry D., (2002), Instabilities in liquid metals controlled by constant magnetic field, Part II : horizontal magnetic field, J. Cryst. Growth, 242: 501-510.

[17] Kaddeche S., Henry D. and Ben Hadid H., (2003), Magnetic stabilization of the buoyant convection between infinite horizontal walls with a horizontal temperature gradient, J. Fluid Mech., 480: $185-216$.

[18] Lappa M., (2016), Control of convection patterning and intensity in shallow cavities by harmonic vibrations, Microgravity Science \& Technology, 28(1): 29-39.

[19] Delgado-Buscalioni R., Crespo del Arco E. And Bontoux P., (2001), Flow transitions of a lowPrandtl-number fluid in an inclined 3D cavity, Eur. J. Mech. B/Fluids, 329: 1-17.

[20] El Ganaoui M., Lamazouade A., Bontoux P. and Morvan D., (2002), Computational solution for fluid flow under solid/liquid phase change conditions, Computers \& Fluids, 31(4-7): 539-556.

[21] Le Quéré P. and Gobin D., (1999), A note on possible flow instabilities in melting from the side, Int. J. Thermal Sciences, 38(7): 595-600.

[22] Hong B., Armaly B.F., Chen T.S., (1993), Laminar mixed convection in a duct with a backward-facing step-the effects of inclination angle and prandtl number, Internat. J. Heat Mass Trans. 36: 3059-3067.

[23] Abu-Mulaweh H. I., Armaly B. F., and Chen T. S., (1996) Laminar natural convection flow over a vertical forward-facing step, Journal of Thermophysics and Heat Transfer, 10(3): 517-523.

[24] Abu-Mulaweh H.I., (2002), Effects of backward- and forward-facing steps on turbulent natural convection flow along a vertical flat plate, International Journal of Thermal Sciences, 41(4): 376385.

[25] Abu-Mulaweh H.I., (2003), A review of research on laminar mixed convection flow over backward- and forward-facing steps, International Journal of Thermal Sciences, 42(9): 897-909.

[26] Abu-Mulaweh H.I., (2005), Turbulent mixed convection flow over a forward-facing step-the effect of step heights, International Journal of Thermal Sciences, 44(2): 155-162

[27] Meskini A., Najam M. and El Alami M., (2011), Convective Mixed Heat Transfer in a Square Cavity with Heated Rectangular Blocks and Submitted to a Vertical Forced Flow, Fluid Dyn. Mater. Process., 7(1): 97-110. 
[28] Dihmani N., Amraqui S., Mezrhab A. and Laraqi N., (2012), Numerical Modelling of Rib Width and Surface Radiation Effect on Natural Convection in a Vertical Vented and Divided Channel, Fluid Dyn. Mater. Process.,. 8(3): 311-322

[29] Mahrouche O., Najam M., El Alami M., Faraji M., (2013), Mixed Convection Investigation in an Opened Partitioned Heated Cavity, Fluid Dyn. Mater. Process., 9(3): 235-250

[30] Kuo H.P., Korpela S.A., (1988), Stability and finite amplitude natural convection in a shallow cavity with insulated top and bottom and heated from the side, Phys. Fluids, 31: 33-42.

[31] Gelfgat A.Yu., Bar-Yoseph P.Z. and Yarin A.L., (1999), Stability of Multiple Steady States of Convection in Laterally Heated Cavities, J. Fluid Mech., 388: 315-334.

[32] Lappa M., (2016), Patterning behaviour of gravitationally modulated supercritical Marangoni flow in liquid layers, Phys. Rev. E, 93(5), 053107, (13 pages).

[33] Kuhlmann H.C. and Nienhüser Ch., (2002), Dynamic free-surface deformations in thermocapillary liquid bridges, Fluid Dynamics Res., 31(2): 103-127.

[34] Burguete J., Mukolobwiez N., Daviaud N., Garnier N., Chiffaudel A., (2001), Buoyantthermocapillary instabilities in extended liquid layers subjected to a horizontal temperature gradient, Phys. Fluids, 13 (10): 2773-2787.

[35] Golovin, A. A., Nepomnyashchy, A. A., and Pismen, L. M. (1997). Nonlinear evolution and secondary instabilities of Marangoni convection in a liquid-gas system with deformable interface. Journal of Fluid Mechanics, 341: 317-341.

[36] Borcia, R., and Bestehorn, M. (2003). Phase-field model for Marangoni convection in liquidgas systems with a deformable interface. Physical Review E, 67(6), 066307.

[37] Fletcher C.A.J., (1991), Computational techniques for fluid-dynamics, (Springer Verlag, Berlin, 1991).

[38] Gresho P. M., Gartling D. K., Torczynski J. R., Cliffe K. A., Winters K. H., Garratt T. J., Spence A. and Goodrich J.W., (1993), is the steady viscous incompressible two-dimensional flow over a backward-facing step at $\mathrm{Re}=800$ stable?, Int. J. Numer. Meth. Fluids 17: 501-541.

[39] Griffith M. D., Thompson M. C., Leweke T., Hourigan K., and Anderson W. P., (2007). Wake behaviour and instability of flow through a partially blocked channel, J. Fluid Mech., 582: 319-340.

[40] Viera F., and Grimshaw R., (1994), Topographic forcing of coastal mesoscale phenomena: Filamentation, vortex formation, and eddy detachment, Journal of physical oceanography, 24(7): 1433-1448.

[41] Boghosian M. E., and Cassel K. W., (2013), A pressure-gradient mechanism for vortex shedding in constricted channels, Physics of Fluids, 25(12), 123603.

[42] Boghosian M. E., and Cassel K. W., (2016), On the origins of vortex shedding in twodimensional incompressible flows, Theoretical and computational fluid dynamics, 30(6): 511-527.

[43] Gharib, M., Rambod, E., Shariff, K., (1998), A universal time scale for vortex ring formation, J. Fluid Mech., 360: 121-140.

[44] Kaiktsis L., Karniadakis G., and Orszag S., (1996), Unsteadiness and convective instabilities in two-dimensional flow over a backward-facing step, J. Fluid Mech., 321: 157-187. 
[45] Wee D., Yi T., Annaswamy A., and Ghoniem A. F., (2004), Self-sustained oscillations and vortex shedding in backward-facing step flows: Simulation and linear instability analysis, Phys. Fluids, 16(9): 3361-3373.

[46] Okano Y., Itoh M. and Hirata A., (1989), Natural and Marangoni Convections in a TwoDimensional Rectangular Open Boat, Journal of Chemical Engineering, 22(3): 275-281.

[47] Okano Y., Umemura S., Enomoto Y., Hayakawa Y., Kumagawa M., Hirata A., Dost S., Numerical study of Marangoni convection effect on the melting of GaSb/InSb/GaSb, J. Cryst. Growth, 235(1-4): 135-139.

[48] Shevtsova V.M., Nepomnyashchy A.A. and Legros J.C., (2003), “Thermocapillary-buoyancy convection in a shallow cavity heated from the side", Phys. Rev. E 67, 066308 (14 pages).

[49] Ben Hadid H. and Roux B., (1989), Buoyancy and thermocapillary driven flows in a shallow open cavity: unsteady flow regimes, J. Cryst Growth, 97(1): 217-225.

[50] Ben Hadid H. and Roux B., (1992), Buoyancy and thermocapillary-driven flows in differentially heated cavities for low Prandtl number fluids, J. Fluid Mech., 235: 1-36.

[51] Mundrane M. and Zebib A., (1994), Oscillatory buoyant thermocapillary flow, Phys. Fluids, 10: 3294-3305.

[52] Gershuni G.Z., Laure P., Myznikov V.M., Roux B., Zhukhovitsky E.M., (1992), On the stability of plane-parallel advective flows in long horizontal layers, Microgravity Q., 2(3): 141-151. [53] Henry D., Kaddeche S. and Ben Hadid H., (2005), Stabilization of thermogravitational flows by magnetic field and surface tension, Phys. Fluids, 17, 054106-1-054106-9.

[54] Parmentier P.M., Regnier V.C. and Lebon G., (1993), Buoyant-thermocapillary instabilities in medium-Prandtl number fluid layers subject to a horizontal temperature gradient, Int. J. Heat Mass Transfer, 36: 2417-2427. 